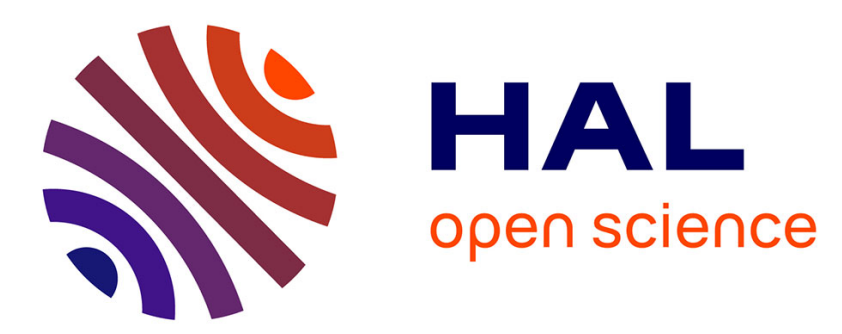

\title{
PDV-based estimation of ejecta particles' mass-velocity function from shock-loaded tin experiment
}

Jean Eloi Franzkowiak, G. Prudhomme, Patrick Mercier, S. Lauriot, E. Dubreuil, Laurent Berthe

\section{- To cite this version:}

Jean Eloi Franzkowiak, G. Prudhomme, Patrick Mercier, S. Lauriot, E. Dubreuil, et al.. PDV-based estimation of ejecta particles' mass-velocity function from shock-loaded tin experiment. Review of Scientific Instruments, 2018, 89, pp.033901 (2018). 10.1063/1.4997365 . hal-02163209

\section{HAL Id: hal-02163209 \\ https://hal.science/hal-02163209}

Submitted on 24 Jun 2019

HAL is a multi-disciplinary open access archive for the deposit and dissemination of scientific research documents, whether they are published or not. The documents may come from teaching and research institutions in France or abroad, or from public or private research centers.
L'archive ouverte pluridisciplinaire HAL, est destinée au dépôt et à la diffusion de documents scientifiques de niveau recherche, publiés ou non, émanant des établissements d'enseignement et de recherche français ou étrangers, des laboratoires publics ou privés. 


\title{
PDV-based estimation of ejecta particles' mass-velocity function from shock-loaded tin experiment
}

\author{
J.-E. Franzkowiak, ${ }^{1,2, a)}$ G. Prudhomme,${ }^{1}$ P. Mercier, ${ }^{1}$ S. Lauriot, ${ }^{1}$ E. Dubreuil, ${ }^{1}$ and L. Berthe ${ }^{2}$ \\ ${ }^{1}$ CEA, DAM, DIF, F-91297 Arpajon, France \\ ${ }^{2}$ PIMM, UMR 8006 CNRS-Arts et Métiers ParisTech, 151 bd de l'Hôpital, F-75013 Paris, France
}

\begin{abstract}
A metallic tin plate with a given surface finish of wavelength $\lambda \simeq 60 \mu \mathrm{m}$ and amplitude $h \simeq 8 \mu \mathrm{m}$ is explosively driven by an electro-detonator with a shock-induced breakout pressure $P_{\mathrm{SB}}=28 \mathrm{GPa}$ (unsupported). The resulting dynamic fragmentation process, the so-called "micro-jetting," is the creation of high-speed jets of matter moving faster than the bulk metallic surface. Hydrodynamic instabilities result in the fragmentation of these jets into micron-sized metallic particles constituting a self-expanding cloud of droplets, whose areal mass, velocity, and particle size distributions are unknown. Lithium-niobate-piezoelectric sensor measured areal mass and Photonic Doppler Velocimetry (PDV) was used to get a time-velocity spectrogram of the cloud. In this article, we present both experimental mass and velocity results and we relate the integrated areal mass of the cloud to the PDV power spectral density with the assumption of a power law particle size distribution. Two models of PDV spectrograms are described. The first one accounts for the speckle statistics of the spectrum and the second one describes an average spectrum for which speckle fluctuations are removed. Finally, the second model is used for a maximum likelihood estimation of the cloud's parameters from PDV data. The estimated integrated areal mass from PDV data is found to agree well with piezoelectric results. We highlight the relevance of analyzing PDV data and correlating different diagnostics to retrieve the physical properties of ejecta particles.
\end{abstract}

\section{INTRODUCTION}

The fragmentation of a roughened metallic plate under shock-loading has been extensively investigated in the last decade, theoretically ${ }^{1-6}$ and experimentally. ${ }^{7-13}$ Many physical mechanisms have to be taken into account: spalling, microspalling, melting, and micro-jetting being the main ones. The increasing interest to characterize these surface destruction products has been leading to many developments, such as $\mathrm{X}$-ray shadowgraphy, piezoelectric pins, Asay foils ${ }^{14}$ Miesizing diagnostic, Photonic Doppler Velocimetry (PDV), ${ }^{15}$ and holography. The purpose is to determine the areal mass of ejecta, the particle sizes, and their velocities. In this article, we focus on the fastest ejecta particles obtained from shockwave loading at a metal vacuum tin interface with a particular surface finish $\left(P_{\mathrm{SB}}=28 \mathrm{GPa}\right)$. We describe a PDV simulation model to analyze time-velocity spectrograms of ejecta clouds. Finally, we show that experimental PDV results can be related to simultaneous piezoelectric measurements through numerical investigations of PDV spectra and mass-velocity distributions, in line with experimental results.

A standard PDV setup, ${ }^{15}$ presented in Fig. 1, has been implemented in our experiment. It consists in mixing two different frequencies to get a beat frequency $\Delta f$ related to the velocity $V$ of the object. Moving particles are illuminated with a single mode continuous-wave fiber laser at $\lambda=1.55 \mu \mathrm{m}$ using a fiber collimator (PDV probe). Doppler-shifted wavelets are collected in the same collimator and mixed with a reference

a)Email: jean-eloi.franzkowiak@cea.fr beam $(\lambda=1.55 \mu \mathrm{m}$, i.e., no frequency up-shifting $)$. For a single moving object, the interference results in a time-beating intensity $I(t)$ on the photodetector whose beat frequency $\Delta f$ can be related to $V$ using the formula $V=\frac{\lambda}{2} \Delta f$,

$$
I(t)=\alpha_{\mathrm{S}} I_{\mathrm{S}}+\alpha_{\mathrm{r}} I_{\mathrm{r}}+2 \sqrt{\alpha_{\mathrm{r}} \alpha_{\mathrm{S}} I_{\mathrm{S}} I_{\mathrm{r}}} \cos (2 \pi \Delta f t+\phi) .
$$

$I_{\mathrm{S}}$ and $I_{\mathrm{r}}$ are, respectively, the beam intensities of the backscattered signal and of the reference signal. $\alpha_{\mathrm{r}}$ and $\alpha_{\mathrm{s}}$ are the coupling parameters of the PDV setup and $\phi$ is the phase difference between reference and backscattered waves.

For a large number of particles with different velocities, the backscattered electric field $E_{\mathrm{s}}(t)$ is the sum of each contribution with its given amplitude $E_{\mathrm{j}}$, frequency $\omega_{j}$, and phase $\phi_{j}$,

$$
E_{\mathrm{S}}(t)=\sum_{\mathrm{j}} E_{\mathrm{j}} \exp \left[i\left(\omega_{\mathrm{j}} t+\phi_{\mathrm{j}}\right)\right] .
$$

Assuming a random particle arrangement (i.e., uncorrelated positions and phases $\phi_{\mathrm{j}}$ uniformly distributed between 0 and $2 \pi$ ), the resulting intensity $I_{\mathrm{s}}(t) \propto\left|E_{\mathrm{S}}(t)\right|^{2}$ has a speckle structure $^{16}$ in both time and frequency (the amplitude and the phase are treated as random variables). The Power Spectral Density (PSD), or the so-called PDV spectrogram, is the squared modulus of the Short-Term Fourier Transform (STFT) of $I(t)$ given by Eq. (1). Recently, Andriyash et al. ${ }^{17}$ studied the influence of the multiple scattering of light on PDV spectra, based on a two-flux solution of the Radiative Transfer Equation (RTE) in the ejecta cloud. They applied this model to analyze the properties of densely packed debris $(\sim 30 \mu \mathrm{m}$ size $)$ with relatively slow velocity gradients (between 100 and $500 \mathrm{~m} / \mathrm{s}$ ). 


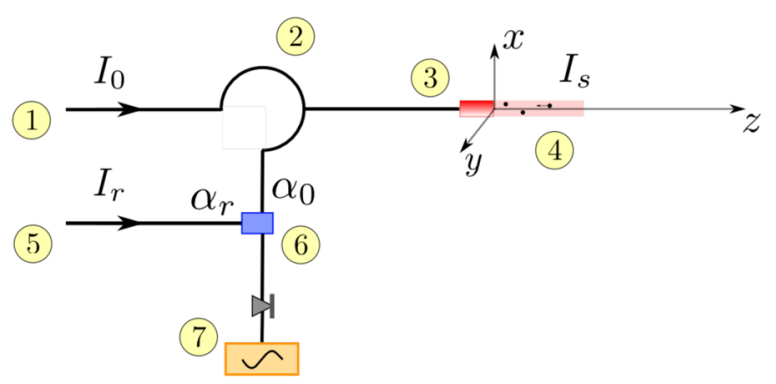

FIG. 1. Standard PDV setup. 1: Laser (beam intensity $I_{0}$ ), 2: optical circulator, 3: optical collimator, 4: moving particles, 5: reference (beam intensity $I_{r}$ ), 6: optical coupler, 7: photodetector and digitizer.

In this article, we study the properties of ejecta clouds with typical particle sizes between 1 and $10 \mu \mathrm{m}$ and velocity gradients around $1500 \mathrm{~m} / \mathrm{s}$. We develop two complementary models of PDV spectra for sparse ejecta cloud using an assumption on the ejecta size distribution (based on holographic measurements ${ }^{18}$ ). We show that the integrated areal mass-velocity distribution estimated from PDV data is in line with piezoelectric experimental results, making possible to solve the inverse problem of determining the areal mass of ejecta with a non-invasive optical diagnostic.

\section{SIMULATION OF PDV SPECTRA}

In Secs. II A and II B, two models of PDV spectra for high-speed ejecta particles are presented.

\section{A. A general simulation of PDV spectrograms}

The physical properties of ejecta cloud and their influence on PDV spectra will be detailed. If the particles are dragging in a gas, the PDV spectrum is time-dependent. ${ }^{19-21}$ Metallic particles ejected in a vacuum will be considered in this article, leading to a statistical time-invariant spectrum if the PDV probe's efficiency does not depend on the viewing distance. In its most general description, the digitized signal $U_{s}(t)$ (in volts) supplied by a collection of $N$ spherical particles depends on many parameters: the incoming gaussian irradiance $\mathcal{E}_{\text {inc }}(r, z)$ $\left(\mathrm{W} / \mathrm{m}^{2}\right)$, the probe's efficiency $\eta(r, z)$, the system's response $R_{\text {sys }}(\mathrm{V} / \mathrm{W})$, the coupling parameters and the reference intensity [respectively, $\alpha_{\mathrm{r}}, \alpha_{\mathrm{s}}, I_{\mathrm{r}}(\mathrm{W})$ ], the volume fractions inside the cloud (volume of matter divided by occupied volume), directly related to the size-velocity distribution, and finally the phase $\phi_{j}$ and the velocity $V_{j}(\mathrm{~m} / \mathrm{s})$ of each particle. The noiseless expression of the raw PDV signal, corresponding to the "AC" part of the signal (digitizers are usually AC coupled so DC terms can be safely ignored), is, for a system of $N$ particles,

$$
\begin{aligned}
U_{s}(t)= & 2 R_{\mathrm{sys}} \sqrt{\alpha_{\mathrm{s}} \alpha_{\mathrm{r}} I_{\mathrm{r}}} \times \mathcal{R} e\left[\sum_{\mathrm{j}=1}^{N} \sqrt{\eta\left(r_{\mathrm{j}}, z_{\mathrm{j}}(t)\right) \mathcal{E}_{\mathrm{inc}}\left(r_{\mathrm{j}}, z_{\mathrm{j}}(t)\right)}\right. \\
& \left.\times \exp \left[-2 \int_{z_{\min }(t)}^{z_{\mathrm{j}}(t)} \frac{1}{l_{\mathrm{ext}}(t, z(t))} d z\right] \cdot \exp \left(2 i k_{0} V_{\mathrm{j}} t+\phi_{\mathrm{j}}\right)\right],
\end{aligned}
$$

where $k_{0}(1 / \mathrm{m})$ is the wave vector at $\lambda=1.55 \mu \mathrm{m}$ and $z_{\min }(t)$ corresponds to the head of the cloud at time $t$ (position of the fastest particle). $l_{\text {ext }}(t, z(t))$ is the extinction length of a slab of particles between $z(t)$ and $z(t)+\delta z(t)$, depending on the particle sizes in the slab and on the volume fraction in particles. $\mathcal{R} e$ is the real part.

The efficiency $\eta(r, z)$ depends on both particle's position and particle's characteristics [size, shape, complex refractive index, and backscattering efficiency $Q_{\mathrm{bs}}\left(d_{\mathrm{p}}\right)$ defined by the radar hypothesis in Ref. 22]. In the following, only spherical particles will be considered.

In Eq. (3), $\mathcal{E}_{\text {inc }}\left(r_{\mathrm{j}}, z_{\mathrm{j}}(t)\right)$ is the gaussian distribution of the irradiance received by each particle at position $\left(r_{\mathrm{j}}, z_{\mathrm{j}}(t)\right.$ ) (see Fig. 2). The negative exponential accounts for the attenuation by scattering (in the forward and backward directions). Some assumptions must be made to write Eq. (3):

- A first order solution of light multiple scattering in the ejecta cloud. A scatter-induced attenuation of the beam is taken into account and multiply scattered photons are neglected, which is valid for dilute systems.

- Velocities and particle sizes are uncorrelated. This may only be true ${ }^{23}$ for the high-velocity region of the spectrum (region visible to PDV). For the estimation of the optical extinction in each slab, a mean particle diameter $\overline{\mathbf{d}_{\mathrm{p}}}$ and a mean extinction efficiency $\overline{\mathbf{Q}_{\mathrm{ext}}}$ will be inferred from the size distribution. Based upon previous results obtained by Sorenson et al. ${ }^{18}$ a power law dependence with a specific exponent $\gamma_{\mathrm{d}}$ is assumed,

$$
f_{\mathrm{d}}\left(d_{\mathrm{p}}\right) \propto d_{\mathrm{p}}^{-\gamma_{\mathrm{d}}} \text {. }
$$

We define an effective volume $\overline{\mathbf{V}_{\mathrm{p}}}$ and the corresponding diameter $\overline{\mathbf{d}_{\mathrm{p}}}$, determined from $f_{\mathrm{d}}$,

$$
\begin{gathered}
\overline{\mathbf{V}_{\mathrm{p}}}=\left(\int_{d_{\text {min }}}^{d_{\max }} f_{\mathrm{d}}\left(d_{\mathrm{p}}\right) \frac{\pi}{6} d_{\mathrm{p}}^{3} \mathrm{~d} d_{\mathrm{p}}\right) \cdot\left(\int_{d_{\text {min }}}^{d_{\max }} f_{\mathrm{d}}\left(d_{\mathrm{p}}\right) \mathrm{d} d_{\mathrm{p}}\right)^{-1}, \\
\overline{\mathbf{d}_{\mathrm{p}}}=\left[\frac{\left(1-\gamma_{\mathrm{d}}\right)\left(d_{\text {max }}^{4-\gamma_{\mathrm{d}}}-d_{\text {min }}^{4-\gamma_{\mathrm{d}}}\right)}{\left(4-\gamma_{\mathrm{d}}\right)\left(d_{\text {max }}^{1-\gamma_{\mathrm{d}}}-d_{\text {min }}^{1-\gamma_{\mathrm{d}}}\right)}\right]^{-\frac{1}{3}} .
\end{gathered}
$$

$d_{\min }$ and $d_{\max }(\mu \mathrm{m})$ are the minimum and maximum particle diameters. An effective particle extinction efficiency $\overline{\mathbf{Q}_{\mathrm{ext}}}$

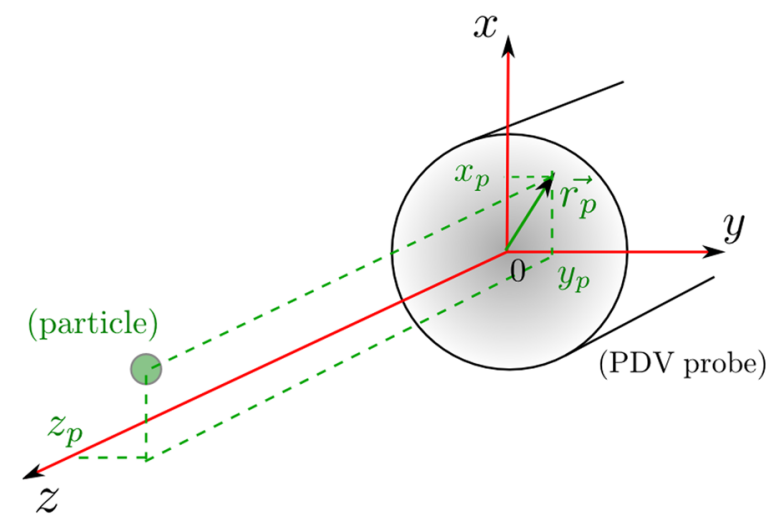

FIG. 2. Coordinates $\left(r_{\mathrm{p}}, z_{\mathrm{p}}\right)$ of a particle in 3D-space with respect to the PDV probe. 
is defined similarly,

$\overline{\mathbf{Q}_{\text {ext }}}=\left(\int_{d_{\text {min }}}^{d_{\text {max }}} Q_{\text {ext }}\left(d_{\mathrm{p}}\right) f_{\mathrm{d}}\left(d_{\mathrm{p}}\right) \mathrm{d} d_{\mathrm{p}}\right) \cdot\left(\int_{d_{\text {min }}}^{d_{\max }} f_{\mathrm{d}}\left(d_{\mathrm{p}}\right) \mathrm{d} d_{\mathrm{p}}\right)^{-1}$.

In Eqs. (5) and (7), $f_{\mathrm{d}}$ is the particle size distribution and $Q_{\text {ext }}\left(d_{\mathrm{p}}\right)$ is the extinction efficiency of a spherical particle calculated using the Mie theory of scattering. The cloud being inhomogeneous along $z$ due to the velocity gradient, we decide to split it into $\mathcal{N}_{\mathrm{s}}$ slabs of identical volume $\mathcal{V}_{\mathrm{s}}(t)$. In each particle slab, the volume fraction $f_{\mathrm{vol}}(z(t), t)$ is estimated at each time $t$. We make the assumption that in each slab, the number of particles is large $(N(z, z+\delta z) \gg 1)$ and the volume of matter in a slab is approximated by $N(z, z+\delta z) \overline{\mathbf{V}_{\mathrm{p}}}$. As the cloud expands in a vacuum, the mesh evolves similarly such that each slab of particles keeps the same amount of matter,

$$
f_{\mathrm{vol}}(z(t), t)=\frac{N(z(t), z(t)+\delta z(t)) \overline{\mathbf{V}_{\mathrm{p}}}}{\mathcal{V}_{\mathrm{s}}(t)} .
$$

The extinction length in Eq. (3), using the first order solution of light scattering, is written as

$$
l_{\mathrm{ext}}(t, z(t))=\frac{2 \overline{\mathbf{d}_{\mathrm{p}}}}{3 f_{\mathrm{vol}}(z(t), t) \overline{\mathbf{Q}_{\mathrm{ext}}}} .
$$

To get an estimation of the collection efficiency $\eta(r, z)$, i.e., the optical power coupled in the system, we detail the calculation for a fixed single particle of coordinates $\left(r_{\mathrm{p}}, z_{\mathrm{p}}\right)$. Using the radar hypothesis, for a particle in the far field of the entrance pupil of the probe (lens), the scattered electric field $E_{\mathrm{s}}$ impinging on the lens is given by the following relationship:

$$
E_{\mathrm{s}}(r, z=0)=\frac{d_{\mathrm{p}}}{4 r} \sqrt{\mathcal{E}_{\mathrm{inc}}\left(r_{\mathrm{p}}, z_{\mathrm{p}}\right) Q_{\mathrm{bs}}\left(d_{\mathrm{p}}\right)} \exp \left(-i k_{0} r\right)
$$

with $r=\sqrt{\left(x-x_{\mathrm{p}}\right)^{2}+\left(y-y_{\mathrm{p}}\right)^{2}+z_{\mathrm{p}}^{2}}$.

The optical power coupling efficiency $\eta\left(r_{\mathrm{p}}, z_{\mathrm{p}}\right)$ is the scalar product of the field scattered by the particle in the entrance pupil with the back-propagated gaussian mode of the fiber $G_{0}(r)$ in the same plane,

$$
\eta\left(r_{\mathrm{p}}, z_{\mathrm{p}}\right)=\left|\int_{\text {pupil }} E_{\mathrm{s}}^{*}(r, z=0) G_{0}(r) d r\right|^{2} \times \frac{1}{\mathcal{E}_{\text {inc }}\left(r_{\mathrm{p}}, z_{\mathrm{p}}\right)} .
$$

For each distance $z$ between the probe and the particle, a mean coupling efficiency $\bar{\eta}(z)$ is obtained $\left[\eta\left(r_{\mathrm{p}}, z_{\mathrm{p}}\right)\right.$ is averaged over a disk of radius $\Phi_{\mathrm{P}} / 2$ located at a distance $z_{\mathrm{p}}$ from the probe with $\Phi_{\mathrm{P}}$ the width of the beam],

$$
\eta(r, z) \mathcal{E}_{\mathrm{inc}}(r, z)=\bar{\eta}(z) \mathcal{E}_{\mathrm{u}}(z),
$$

where $\mathcal{E}_{\mathrm{u}}(z)\left(\mathrm{W} / \mathrm{m}^{2}\right)$ is the equivalent uniform irradiance. Given a discrete number of particles whose positions and velocities are known at $t=0$ in the mesh, we can construct the raw PDV signal at each time step and obtain the PDV spectrum $\Phi(V, t)$ by computing a conventional STFT.

An example of such a PDV spectrogram $\Phi(V, t)$ is shown in Fig. 3. We observe the typical time-varying speckle fluctuations coming from the interferences between the Dopplershifted wavelets and the reference beam. For neighboring particles having close velocities, their velocity traces are not discriminated from the Fourier analysis and a single particle velocity cannot be extracted in the spectrogram. On the left, we can observe the evolution of the coherent irradiance inside the cloud versus the penetration distance $z$.

\section{B. Analytical model of PDV spectrograms}

We add new assumptions to get an analytical description of a PDV spectrum for an $N$-particle system expanding in a vacuum. In the case of linear Richtmyer-Meshkov instabilities (for $k h<1$ ) and based on recent molecular dynamics results, ${ }^{5,23-25}$ the integrated mass-velocity distribution can be very well approximated by an exponential function. Consequently, an exponential distribution of velocities will be assumed,

$$
f_{\mathrm{v}}(V)=J_{0} e^{-\gamma_{\mathrm{v}} \frac{\left(V-V_{\min }\right)}{V_{\max }}},
$$
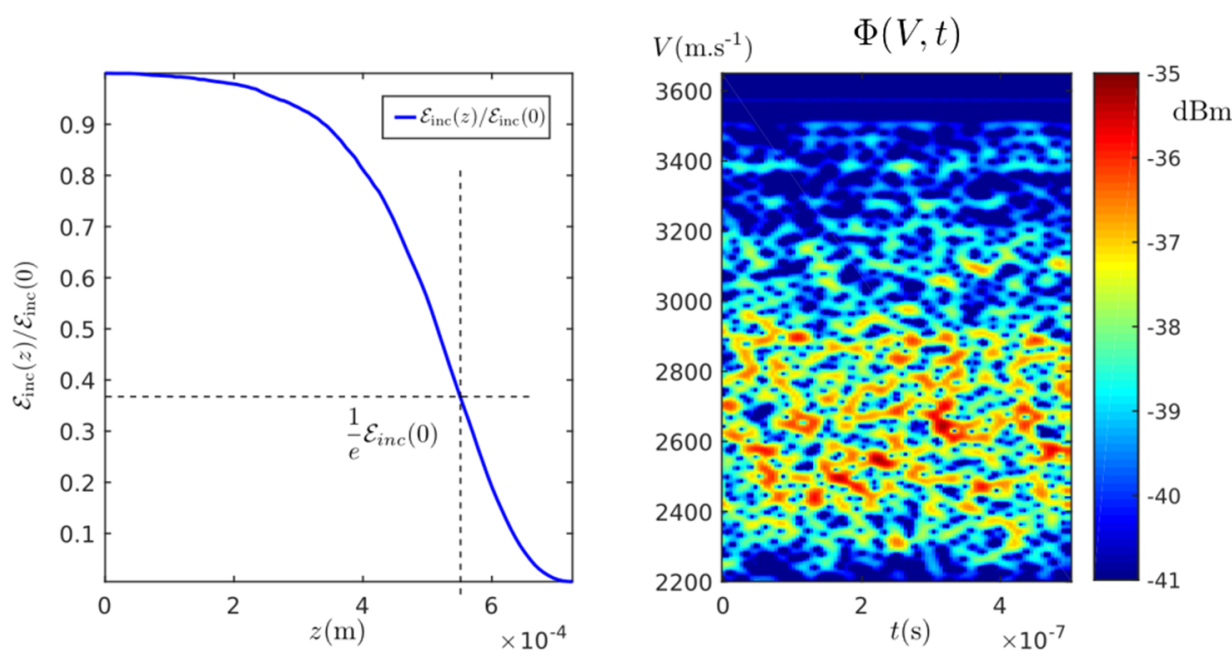

FIG. 3. Noiseless simulated PDV spectrogram $\Phi(V, t)$ (right) and evolution of the irradiance $\mathcal{E}_{\text {inc }}(z)$ inside the cloud (left) from Eq. (3) for a polydisperse expanding cloud in a vacuum at time $t=500 \mathrm{~ns} . z=0$ is the head of the cloud (fastest particles) and $L_{\text {cloud }}=725 \mu \mathrm{m}$. Velocities are exponentially distributed with $\gamma_{\mathrm{v}}=18$ [cf. Eq. (13)], and particle sizes follow a power law distribution with characteristic exponent $\gamma_{\mathrm{d}}=4 . d_{\mathrm{min}}=1 \mu \mathrm{m}, d_{\mathrm{max}}=10 \mu \mathrm{m}, V_{\mathrm{min}}=2200 \mathrm{~m} / \mathrm{s}$, $V_{\max }=3650 \mathrm{~m} / \mathrm{s}$, and $M_{\mathrm{s}}=2 \mathrm{mg} / \mathrm{cm}^{2}$. Collimated optical probe $\left(\omega_{0}=150 \mu \mathrm{m}, z_{\mathrm{f}}=0\right), \phi_{\mathrm{P}}=300 \mu \mathrm{m}, I_{0}=\mathcal{E}_{\text {inc }}(r, 0) \pi \frac{\Phi_{\mathrm{P}}^{2}}{4}=400 \mathrm{~mW}$. [Short-Time Fourier Transform (STFT) parameters: sliding window $F_{\mathrm{w}}$ : Hanning, width $T_{\mathrm{w}}=50 \mathrm{~ns}$, time step $\delta T_{\mathrm{w}}=10 \%$, zero-padding $Z_{\mathrm{p}}=4$.] 
where $J_{0}$ is a multiplicative factor. The integration of $f_{\mathrm{v}}$ between $V_{\min }$ and $V_{\max }$ is equal to the total number of particle $N_{\text {tot }}$,

$$
N_{\text {tot }}=J_{0} \frac{V_{\max }}{\gamma_{\mathrm{v}}}\left[1-e^{-\frac{\gamma_{\mathrm{v}} \Delta V}{V_{\max }}}\right] .
$$

$\Delta V=V_{\max }-V_{\min } . N_{\text {tot }}$ is related to the total areal mass of the cloud $M_{\mathrm{S}}\left(\mathrm{kg} / \mathrm{m}^{2}\right)$ through the relationship

$$
\frac{4 N_{\mathrm{tot}} \overline{\mathbf{V}_{\mathrm{p}} \rho}}{\pi \Phi_{\mathrm{E}}^{2}}=M_{\mathrm{s}} .
$$

$\rho$ is the density of tin $\left(\mathrm{kg} \mathrm{m}^{-3}\right)$ and $\pi \Phi_{\mathrm{E}}^{2} / 4$ is the surface of ejection $\left(\mathrm{m}^{2}\right)$. Finally, $f_{\mathrm{v}}$ is written as

$$
f_{\mathrm{v}}(V)=M_{\mathrm{s}} \frac{\gamma_{\mathrm{v}} \pi \Phi_{\mathrm{E}}^{2}}{4 \overline{\mathbf{V}_{\mathrm{p}}} \rho V_{\max }} \cdot \frac{1}{1-\exp \left[-\gamma_{\mathrm{v}} \frac{\Delta V}{V_{\max }}\right]} \cdot e^{-\gamma_{\mathrm{v}} \frac{\left(V-V_{\min }\right)}{V_{\max }}} .
$$

We add two points in order to precise the model:

- The first point deals with the self-expansion of the cloud that links the coordinates $z$ of the particles to their velocities (no dragging), i.e.,

$$
N(z) \delta z=N(V) \delta V .
$$

- The second point is focused on replacing the backscattering efficiency of each particle with an average efficiency $\overline{\mathbf{Q}_{\mathrm{bs}}}$,

$$
\overline{\mathbf{Q}_{\mathrm{bs}}}=\left(\int_{d_{\text {min }}}^{d_{\text {max }}} Q_{\mathrm{bs}}\left(d_{\mathrm{p}}\right) f_{\mathrm{d}}\left(d_{\mathrm{p}}\right) \mathrm{d} d_{\mathrm{p}}\right) \cdot\left(\int_{d_{\text {min }}}^{d_{\text {max }}} f_{\mathrm{d}}\left(d_{\mathrm{p}}\right) \mathrm{d} d_{\mathrm{p}}\right)^{-1} .
$$

As the cloud rapidly self-expands in a vacuum, the first point is meaningful. The second point is only valid if the statistical properties of the cloud are invariant by translation in the $x$ and $y$ directions and if the total number of particles in each slab is large $(N \gg 1)$.

The average PSD, $\mathcal{P}(V)=\langle\Phi(V, t)\rangle_{t_{\infty}}(\mathrm{W})$, between velocities $V$ and $V+\delta V$, is proportional to the average intensity scattered in the velocity range $[V, V+\delta V]$. Position being related to velocity, the spatial integral in the exponential argument [Eq. (3)] can be replaced with an integral over velocities. For a collimated beam, $\mathcal{E}_{\mathrm{u}}(z)=\mathcal{E}_{\mathrm{u}}$. Introducing the velocity distribution $f_{\mathrm{v}}$, the average $\operatorname{PSD}\langle\Phi(V, t)\rangle_{t_{\infty}}$ is

$$
\begin{aligned}
\langle\Phi(V, t)\rangle_{t_{\infty}}= & |\sqrt{\mathcal{P}(V)}|^{2}=\mid \int_{-\infty}^{+\infty} \sqrt{f_{\mathrm{v}}(V) \cdot \bar{\eta} \cdot \mathcal{E}_{\mathrm{u}}} \\
& \times\left.\exp \left[-\frac{3 \overline{\mathbf{Q}_{\mathrm{ext}}} \overline{\mathbf{V}_{\mathrm{p}}}}{\overline{\mathbf{d}_{\mathrm{p}}} \pi \phi_{\mathrm{P}}^{2}} \int_{V}^{V_{\max }} f_{\mathrm{v}}(V) \mathrm{d} V\right] \mathrm{d} V\right|^{2} .
\end{aligned}
$$

To take into account the Fourier window $F_{\text {w }}$ of finite width $T_{\mathrm{w}}$, the upper definition of $\langle\Phi(V, t)\rangle_{t_{\infty}}$ must be modified and a convolution between $F_{\mathrm{w}}$ and $\sqrt{\mathcal{P}(V)}$ must be computed for each velocity $V$,

$$
\langle\Phi(V, t)\rangle_{t_{\infty}}=\left|\sqrt{\mathcal{P}(V)} \otimes F_{\mathrm{w}}(V)\right|^{2} .
$$

For a rectangular window of width $T_{\mathrm{w}}$ and an exponential velocity distribution $f_{\mathrm{v}}(V)$ [Eq. (13)], we finally get for each $V$

$$
\begin{aligned}
\langle\Phi(V, t)\rangle_{t_{\infty}}= & \frac{2 T_{\mathrm{w}}}{\lambda} \mid \int_{-\infty}^{+\infty} \sqrt{\epsilon \cdot \beta M_{\mathrm{s}}} \cdot e^{-\frac{\gamma_{\mathrm{v}}\left(\tilde{V}-V_{\min }\right)}{2 \cdot V_{\max }}} \\
& \cdot \exp \left[-\frac{3 \overline{\mathbf{Q}_{\mathrm{ext}}} \overline{\mathbf{V}_{\mathrm{p}}}}{\overline{\mathbf{d}_{\mathrm{p}}} \pi \phi_{\mathrm{P}}^{2}} \cdot \beta \cdot M_{\mathrm{s}} \cdot \frac{V_{\max }}{\gamma_{\mathrm{v}}}\left[e^{-\gamma_{\mathrm{v}} \frac{\tilde{V}-V_{\min }}{V_{\max }}}\right.\right. \\
& \left.\left.-e^{-\gamma_{\mathrm{v}} \frac{\Delta V}{V_{\max }}}\right]\right]\left.\cdot \operatorname{sinc}\left(\frac{2 T_{\mathrm{w}}}{\lambda}(V-\tilde{V})\right) d \tilde{V}\right|^{2} \cdot
\end{aligned}
$$

$\langle.\rangle_{t_{\infty}}$ is the time average. If we average an infinite number of independent speckle realizations, the fluctuations are removed and the PSD is $\langle\Phi(V, t)\rangle_{t_{\infty}}$, the parameter of the speckle statistics. When the sliding time step of the Fourier window is equal to its width, adjacent points in the PDV spectrogram are independent and time-averaging is equivalent to averaging over different particle cloud realizations (ensemble average). $\epsilon(\mathrm{W})$ is a multiplicative constant accounting for the collection efficiency of the probe $\left(\epsilon=\bar{\eta} \cdot \mathcal{E}_{\mathrm{u}}\right)$ and $\Delta V=V_{\max }-V_{\min }$. The transverse size of the cloud $\phi_{\mathrm{E}}$ is equal to the width of the beam $\phi_{\mathrm{P}} . M_{\mathrm{S}}$ is the total areal mass of the cloud $\left(\mathrm{kg} / \mathrm{m}^{2}\right), \sin \mathrm{c}(x)=\sin (\pi x) / \pi x$, and $\beta(\mathrm{m} \mathrm{s} / \mathrm{kg})$ is given by the following relationship:

$$
\beta=\frac{\gamma_{\mathrm{v}} \pi \Phi_{\mathrm{P}}^{2}}{4 \overline{\mathbf{V}_{\mathrm{p}}} \rho V_{\max }} \cdot \frac{1}{1-\exp \left[-\gamma_{\mathrm{v}} \frac{\Delta V}{V_{\max }}\right]} .
$$

$\langle\Phi(V, t)\rangle_{t_{\infty}}$ can be summed to recover the total optical intensity (W). Equation (21) gives an estimate of the average PSD for a self-expanding particle cloud in a vacuum, whose size and velocity distributions have, respectively, a power law and an exponential dependence. Since the constant $\epsilon$ does not depend on the properties of the cloud, it will be held constant. This will not have any consequence for a relative comparison between different PDV spectra.

In Fig. 4, we can observe the time-averaged PSD (spectrogram of Fig. 3) and the PSD obtained from Eq. (21). The red curve would be obtained if an infinite number of independent realizations (see Fig. 3) were averaged.

With these two models, we get access to a fluctuating PDV spectrogram (model 1) and to what is known as an "average PDV spectrogram" for which the characteristic speckle fluctuations are removed (model 2). Before drawing a direct comparison with experimental results, the way in which the parametric dependencies of the ejecta cloud modify the shape of the spectrum has to be considered [Eq. (21)].

\section{PDV spectrum and parametric dependencies}

The influence of ejecta parametric dependencies on the PDV spectrum will be briefly discussed, based on the calculation of the average spectrogram $\langle\Phi(V, t)\rangle_{\mathrm{t}_{\infty}}$ from Eq. (21) (model 2). We recall that this analysis is only valid for a self-expanding cloud in a vacuum. The integrated areal massvelocity $M(V)$ is the cumulative areal mass $\left(\mathrm{mg} \mathrm{cm}^{-2}\right)$ between $V$ and $V_{\max }$ with $M\left(V_{\min }\right)=M_{\mathrm{s}}$. 

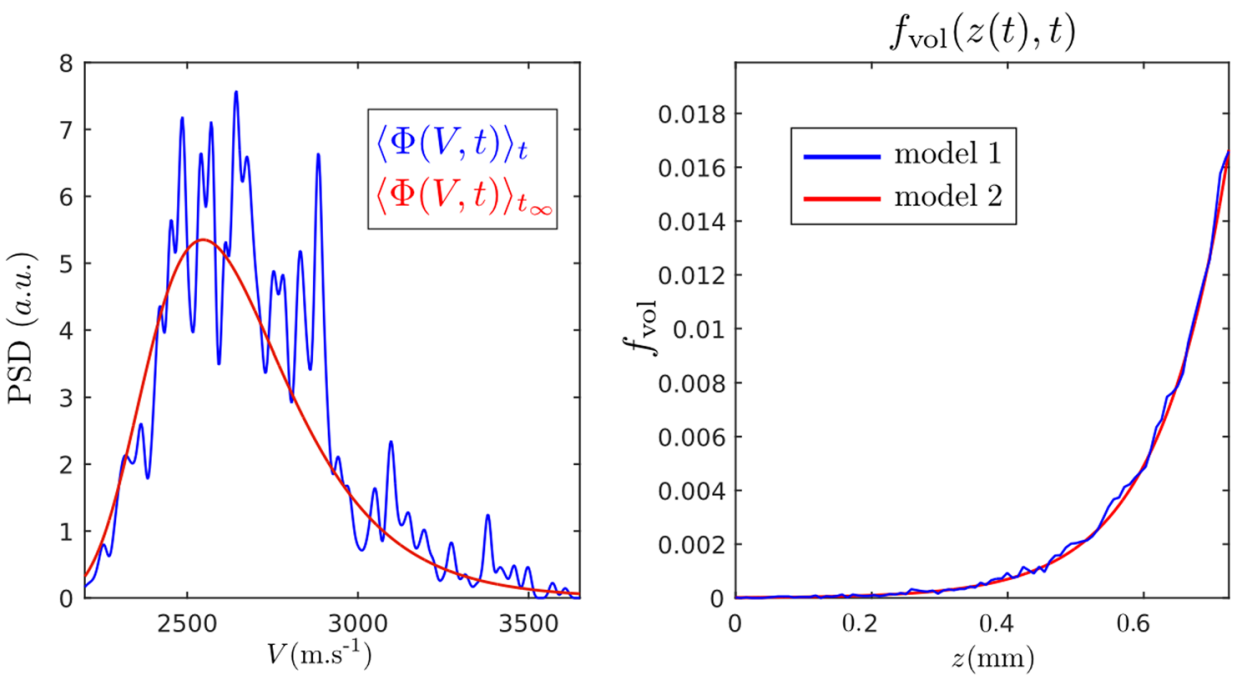

FIG. 4. Comparison between $\langle\Phi(V, t)\rangle_{t}$ averaged over $500 \mathrm{~ns}$, obtained from model 1, and the PDV spectrum $\langle\Phi(V, t)\rangle_{t_{\infty}}$ obtained from model 2 [Eq. (21)] for a polydisperse expanding cloud in a vacuum. The cloud's properties are described in the caption of Fig. 3 . The red curves correspond to Eq. (21) and the blue ones correspond to Eq. (3). On the right are plotted the spatial evolutions of the volume fractions inside the cloud for the 2 models (total length of the cloud $L_{\text {cloud }}=725 \mu \mathrm{m}$ ) at time $t=500 \mathrm{~ns} . z=0$ is the head of the cloud (fastest particles).

\section{Influence of the areal mass $M_{s}$}

The properties of the cloud remain unchanged (size and velocity distributions), except its areal mass $M_{\mathrm{s}}\left(\mathrm{mg} / \mathrm{cm}^{2}\right)$.

The impact of increasing the areal mass on the mean PDV spectrum is shown in Fig. 5. The maxima of the spectrum are shifted to higher velocities. It arises from the fact that particle volume fractions are increasingly densified at high velocities. Two phenomena must be taken into account: the first one is the increased attenuation by scattering due to increased particle densities and the second one is the increase of the mean collected intensity (more particles). The penetration of the beam is large for a small areal mass (see green curves in Fig. 5) and reduced for a larger one (red curves): the PSD drastically falls below $V=2500 \mathrm{~m} / \mathrm{s} .\langle\Phi(V, t)\rangle_{\mathrm{t}_{\infty}}$ remains large at high velocities, where the beam has not yet been impacted by attenuation.

\section{Influence of the velocity distribution}

All properties are still unchanged except the characteristic exponent $\gamma_{\mathrm{v}}$ of the velocity distribution. We recall the form of the velocity distribution $f_{\mathrm{v}}$,

$$
f_{\mathrm{V}}(V) \propto e^{-\gamma_{\mathrm{v}} \frac{\left(V-V_{\min }\right)}{V_{\max }}} .
$$

A change in velocity distribution drastically impacts both slopes and amplitude of the spectrum $\langle\Phi(V, t)\rangle_{t_{\infty}}$. For large values of $\gamma_{\mathrm{v}}$ (see green curves in Fig. 6), the velocity distribution is highly peaked at low velocities and the few high-speed particles (near $3650 \mathrm{~m} / \mathrm{s}$ ) are not detected. The location of the maximum always depends on a compromise between extinction and scattering of light. For small values of $\gamma_{\mathrm{v}}$, particle densities are increased in the high-velocity region and the trade-off between attenuation and scattering appears for higher velocities (around $2750 \mathrm{~m} / \mathrm{s}$ for $\gamma_{\mathrm{v}}=18$ ).

\section{Influence of the size distribution}

We study the influence of the exponent $\gamma_{\mathrm{d}}$ on the PDV spectrum,

$$
f_{\mathrm{d}}\left(d_{\mathrm{p}}\right)=d_{\mathrm{p}}^{-\gamma_{\mathrm{d}}} .
$$

In Fig. 7, we can see the impact of increasing $\gamma_{\mathrm{d}}$. For $\gamma_{\mathrm{d}}=2$, the average particle diameter is larger than for a highly peaked size distribution obtained with $\gamma_{\mathrm{d}}=5.5$. As the areal mass density is kept constant $\left(3 \mathrm{mg} / \mathrm{cm}^{2}\right)$, the number of particles contributing to the amplitude of the spectrum grows with increasing $\gamma_{\mathrm{d}}$. Regarding the smallest particles $\left(\gamma_{\mathrm{d}}=6\right)$, we can also notice that the optical attenuation of light is more
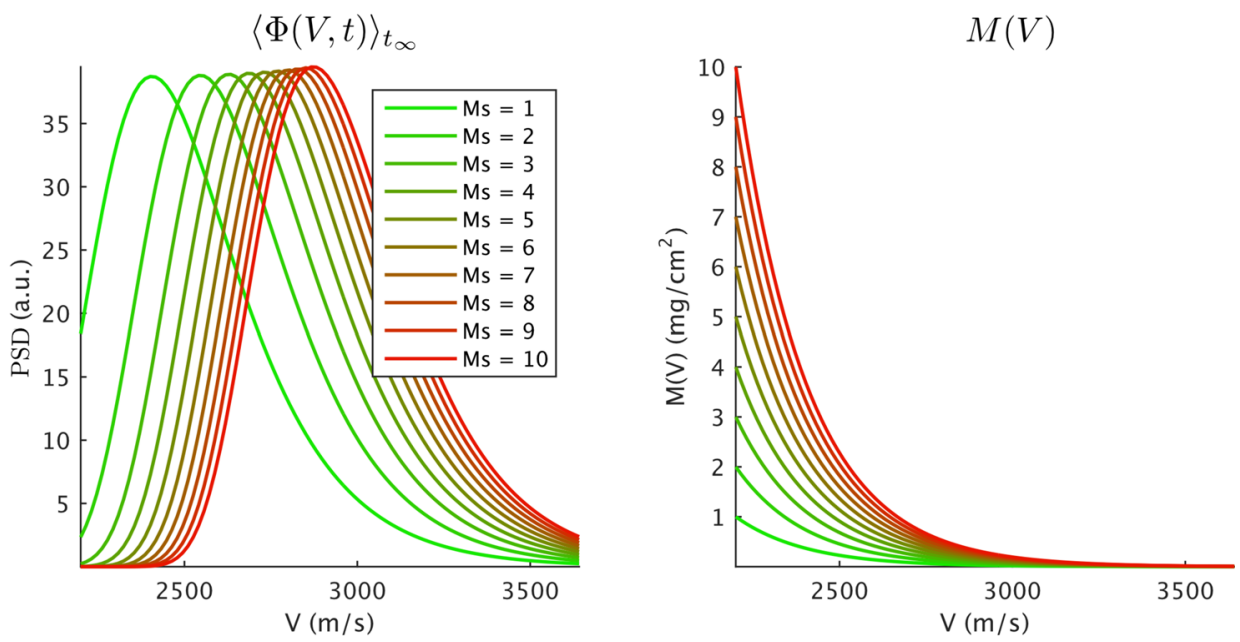

FIG. 5. Influence of the areal mass $M_{s}$ $\left(\mathrm{mg} / \mathrm{cm}^{2}\right)$ on the average PDV spectrum $\langle\Phi(V, t)\rangle_{t_{\infty}}(\mathrm{W})$ and on $M(V)\left(\mathrm{mg} / \mathrm{cm}^{2}\right)$ inside the cloud. $\gamma_{\mathrm{d}}=4, \gamma_{\mathrm{v}}=18, V_{\min }$ $=2200 \mathrm{~m} / \mathrm{s}, V_{\max }=3650 \mathrm{~m} / \mathrm{s}, d_{\min }$ $=1 \mu \mathrm{m}$, and $d_{\max }=10 \mu \mathrm{m}$. 

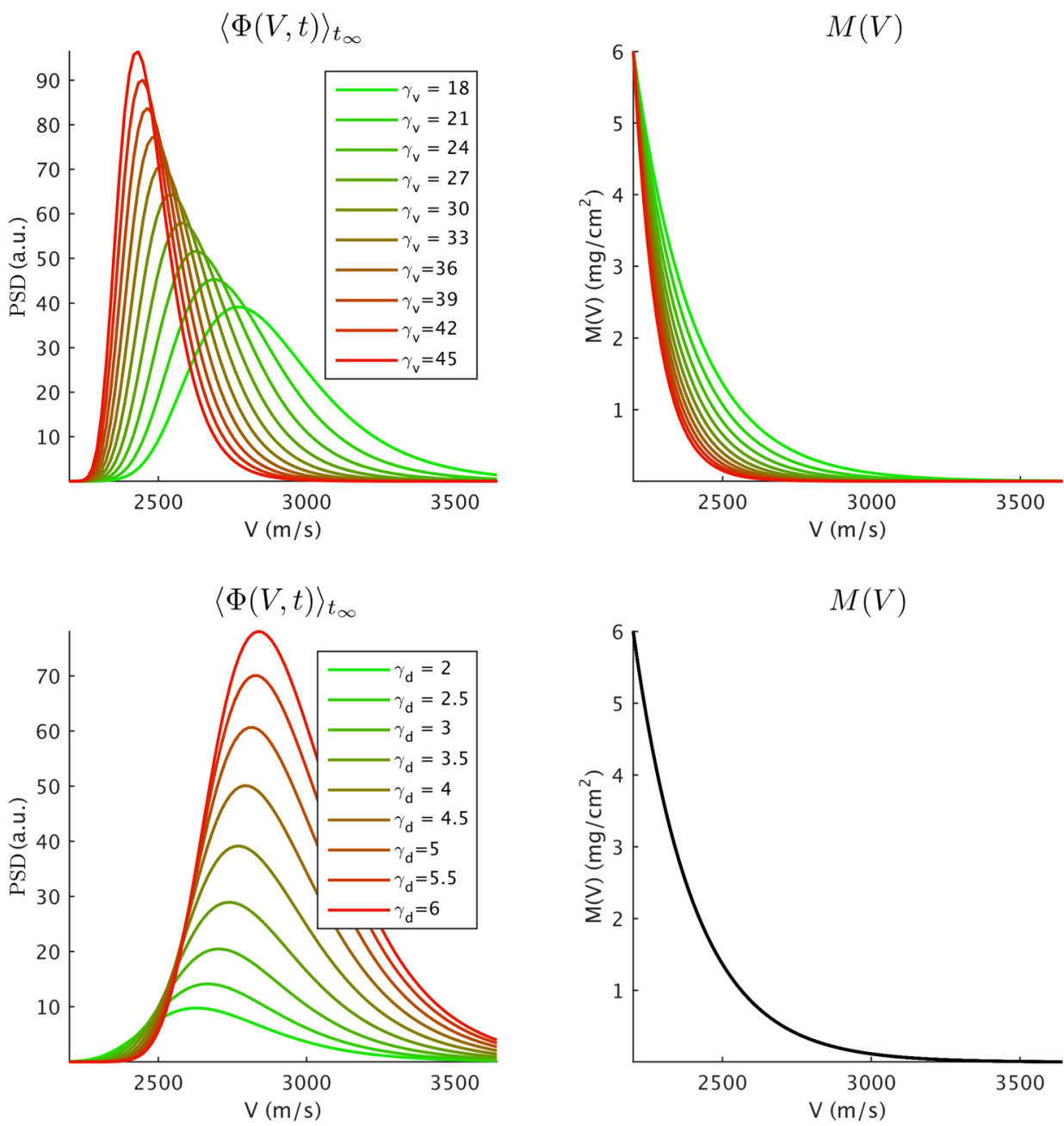

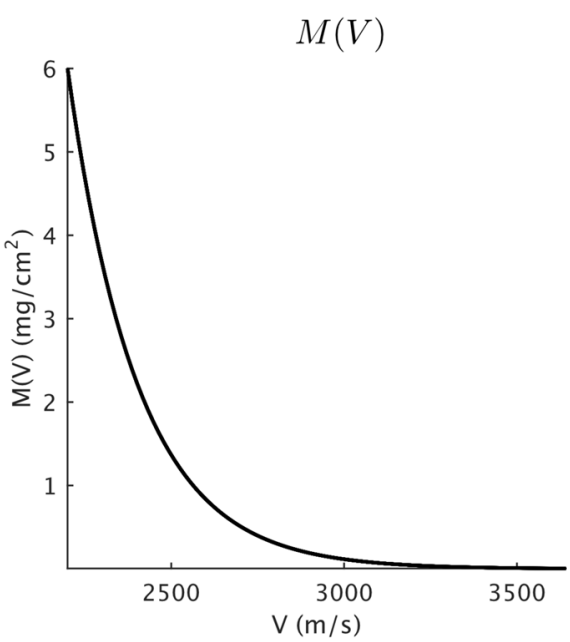

FIG. 6. Influence of velocity distribution $f_{\mathrm{V}}$ on the average PDV spectrum $\langle\Phi(V, t)\rangle_{t_{\infty}}(\mathrm{W})$ and on $M(V)\left(\mathrm{mg} / \mathrm{cm}^{2}\right)$ inside the cloud. $\gamma_{\mathrm{d}}=4, M_{\mathrm{s}}=6 \mathrm{mg} / \mathrm{cm}^{2}$, $V_{\min }=2200 \mathrm{~m} / \mathrm{s}, V_{\max }=3650 \mathrm{~m} / \mathrm{s}$, $d_{\min }=1 \mu \mathrm{m}$, and $d_{\max }=10 \mu \mathrm{m}$.
FIG. 7. Influence of the size distribution $f_{\mathrm{d}}$ on the average PDV spectrum $\langle\Phi(V, t)\rangle_{t_{\infty}}(\mathrm{W})$ and on $M(V)$ $\left(\mathrm{mg} / \mathrm{cm}^{2}\right)$ inside the cloud. $\gamma_{\mathrm{v}}=18$, $M_{\mathrm{s}}=3 \mathrm{mg} / \mathrm{cm}^{2}, V_{\min }=2200 \mathrm{~m} / \mathrm{s}$, $V_{\max }=3650 \mathrm{~m} / \mathrm{s}, d_{\min }=1 \mu \mathrm{m}$, and $d_{\max }=10 \mu \mathrm{m}$. severe and the position of the maximum is shifted to high velocities.

This parametric study is of great interest to understand the influence of the microphysical properties of the cloud on the PDV spectrum. We are aware that a real PDV spectrum is inherently noisy due to the speckle statistics (Fig. 3) and average PDV spectra shown in Figs. 5-7 will never be obtained in any ejecta experiment. However, in the case of a self-expanding ejecta in a vacuum, a relevant time-averaging can be done to mitigate speckle fluctuations and an average model of the PDV spectrum can be used to find the best fit and try to resolve the inverse problem of determining ejecta's properties with non-invasive PDV diagnostic.

\section{EXPERIMENT AND COMPARISONS}

The experimental setup is described below. An electrodetonator is used to generate a shockwave in a $1 \mathrm{~mm}$-thick tin plate. In the first experiment, the free surface of the plate is flat (e.g., diamond turned). For the second one, the surface was finished to get $2 \mathrm{D}$ periodic triangular patterns of width $60 \mu \mathrm{m}$ and height $8 \mu \mathrm{m}$. The distance between the probes and the plate is set to $7 \mathrm{~mm}$. The lateral size of the plate is $4 \mathrm{~mm}$. For the second experiment, the shockwave interaction with the perturbed surface creates metal sheets which fragment into high-speed micron-sized metallic particles in a vacuum.
The unsupported peak pressure at the free surface is approximately $28 \mathrm{GPa}$. The signals were recorded with a sampling frequency of $50 \mathrm{GS} / \mathrm{s}$ (20 ps time step) during several $\mu \mathrm{s}$. We do not focus on the ejection mechanism which has been extensively studied, and this way experimental conditions will be kept constant (shockwave pressure, plate thickness, and surface finish). PDV probes are gradient index (GRIN) lenses with an output beam diameter of $100 \mu \mathrm{m}$ (purchased from IDIL Fibres Optiques, Inc., reference COCOM02472). Despite the fact that the coupling efficiency of this probe has not been precisely defined, our analysis will not be limited since we are interested in relative comparisons between PDV spectrograms. The optical power delivered by the PDV probe is set to $300 \mathrm{~mW}$ at $\lambda=1.55 \mu \mathrm{m}$. The use of piezoelectric probes for areal mass measurements has been demonstrated ${ }^{26-28}$ and we used a standard lithium-niobate (LN) sensor (purchased from Dynasen, Inc.).

\section{A. PDV spectrogram results}

To get an estimate of the free-surface velocity, a first experiment was performed on a flat tin surface of width $1 \mathrm{~mm}$ (the setup is described in Fig. 8). Two frontal PDV probes are located $1.75 \mathrm{~mm}$ off center at $7 \mathrm{~mm}$ from the surface. The shock is not perfectly plane at $1.75 \mathrm{~mm}$ and a correction must be made since PDV measures the line-of-sight velocity vector $V_{\mathrm{z}}$. From a hydrodynamic simulation using CEA 


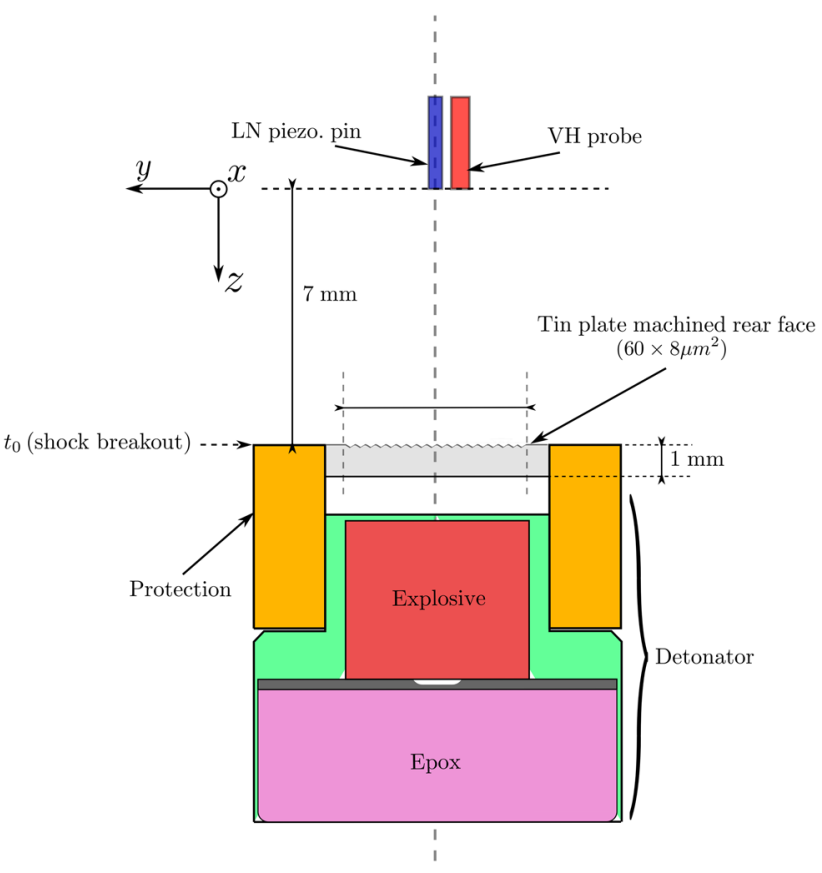

FIG. 8. Experimental setup.

Hesione code, an angle of $\theta \simeq 6^{\circ}$ is found between the freesurface velocity vector $\vec{V}_{\mathrm{fs}}$ and the z-axis such that $V_{\mathrm{fs}} \simeq V_{\mathrm{z}} /$ $\cos \theta=2013 \mathrm{~m} / \mathrm{s}$ (see Fig. 9). This free-surface velocity will be our reference for the following analysis on piezoelectric and PDV data.

In a second experiment, simultaneous frontal PDV and LN piezoelectric probes have been implemented (Fig. 8). The average background noise $\left\langle\mathrm{B}_{\Phi_{\text {exp }}}\right\rangle$ is estimated in a region of the spectrum $\Phi_{\exp }$ (Fig. 10) without signal ( $\left.V \geq 5000 \mathrm{~m} / \mathrm{s}\right)$. Since the probe's collection efficiency is not constant with $z$ (diverging beam), both the fastest and the slowest particles appear at later time, the high optical energy density near the probe increasing the penetration of the beam in the cloud. Around

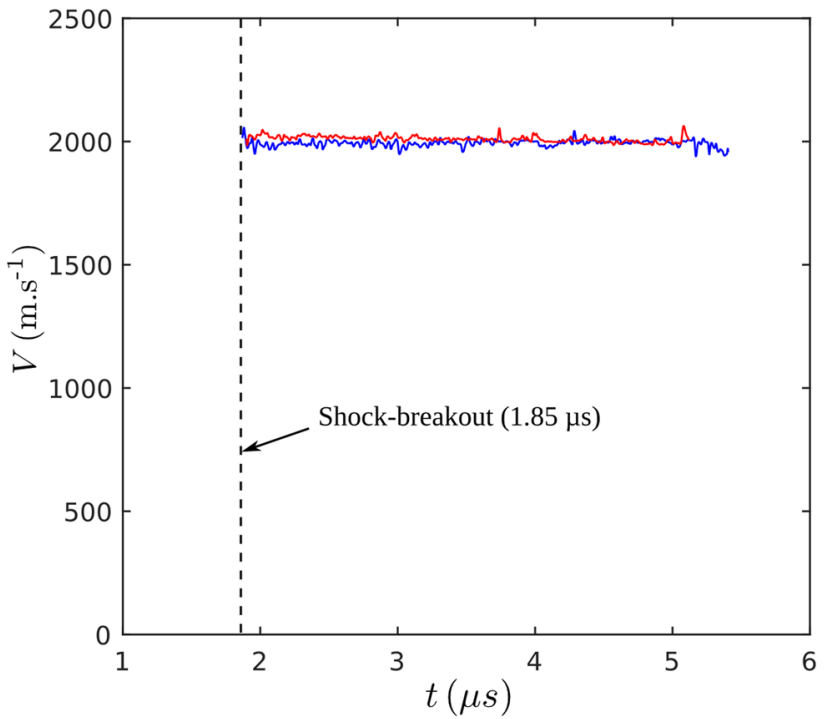

FIG. 9. First experiment: Velocity of an explosively driven $1 \mathrm{~mm}$-thick flat tin surface measured by two frontal PDV probes located at $1.75 \mathrm{~mm}$ off center. The mean velocity is $V_{\mathrm{fs}} \simeq 2013 \mathrm{~m} / \mathrm{s}$ (with an angle correction of $6^{\circ}$ ).

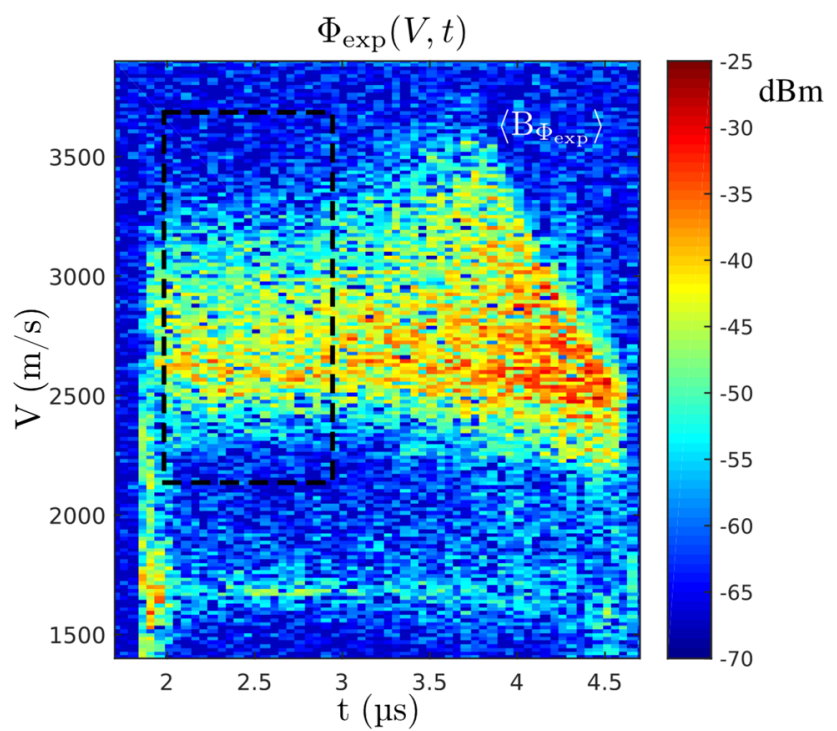

FIG. 10. Second experiment: Experimental PDV spectrogram $\Phi_{\exp }(V, t)$ for a shockwave loaded $1 \mathrm{~mm}$-thick tin plate experiment $(60 \times 8 \mu \mathrm{m}$ grooves $)$ at $P_{\mathrm{SB}} \simeq 28 \mathrm{GPa}$, the configuration being described in Fig. 8. Fourier window: Rectangular, $T_{w}=50 \mathrm{~ns}, \delta T_{w}=100 \%$, no zero-padding. The black dashed box defines the limits of our analysis: time ranges from $t=1.95 \mu$ s to $t=2.9$ $\mu$ s and velocities from $V_{\min }=2281 \mathrm{~m} / \mathrm{s}$ to $V_{\max }=3676 \mathrm{~m} / \mathrm{s}$. $\left\langle B_{\Phi_{\exp }}\right\rangle$ is the average background noise of the PDV spectrogram.

$t \simeq 3.8 \mu \mathrm{s}$, the fastest particles hit the probe, corresponding to a time of flight of approximately $2 \mu$ s for a particle moving at $3500 \mathrm{~m} / \mathrm{s}$. Within the first microsecond, we assume that the statistics of the cloud is unchanged (the collection efficiency of the probe is assumed to be constant) and the data $\Phi_{\exp }(V, t)$ are averaged on a finite interval, between $t=1.95 \mu \mathrm{s}$ and $t=2.9 \mu \mathrm{s}$, to get $\left\langle\Phi_{\exp }(V)\right\rangle_{t}$.

\section{B. Piezoelectric pin results}

The piezoelectric pin used in this experiment was a Lithium-Niobate (LN) y $+36^{\circ}$-cut crystal of diameter $1.27 \mathrm{~mm}$. The compression of the crystal, induced by accumulation of particles and in the absence of any applied electric field, leads to the creation of a charge density $D_{i}$ given by

$$
D_{i}=d_{i j} \sigma_{j},
$$

where $d_{i j}$ is the piezoelectric sensitivity and $\sigma_{j}$ is the applied stress. It will be assumed that the stress is applied in the $\mathrm{z}$ direction ( $i=j$ for uniaxial compression), that all particles are ejected instantaneously at shock breakout $\left(t_{0}\right)$, and that particle collisions are inelastic. The sensitivity of the LN-pin is assumed to be $d_{i i}=24 \mathrm{pC} / \mathrm{N}$ and the fail pressure is around $0.6 \mathrm{GPa}$. The time-dependent voltage response $U(t)$ across an impedance $R(50 \Omega)$ shown in Fig. 11 is linked to the timedependent applied stress $\sigma(t)(\mathrm{GPa})$,

$$
\sigma(t) \simeq \frac{1}{d_{i i} A} \int_{t_{0}}^{t} \frac{U(t)}{R} d t .
$$

The integrated areal mass $M_{a}(t)$ is calculated as follows:

$$
M_{a}(t)=\int_{t_{0}}^{t} \frac{\sigma(t)}{V_{\mathrm{p}}(t)} d t^{\prime},
$$




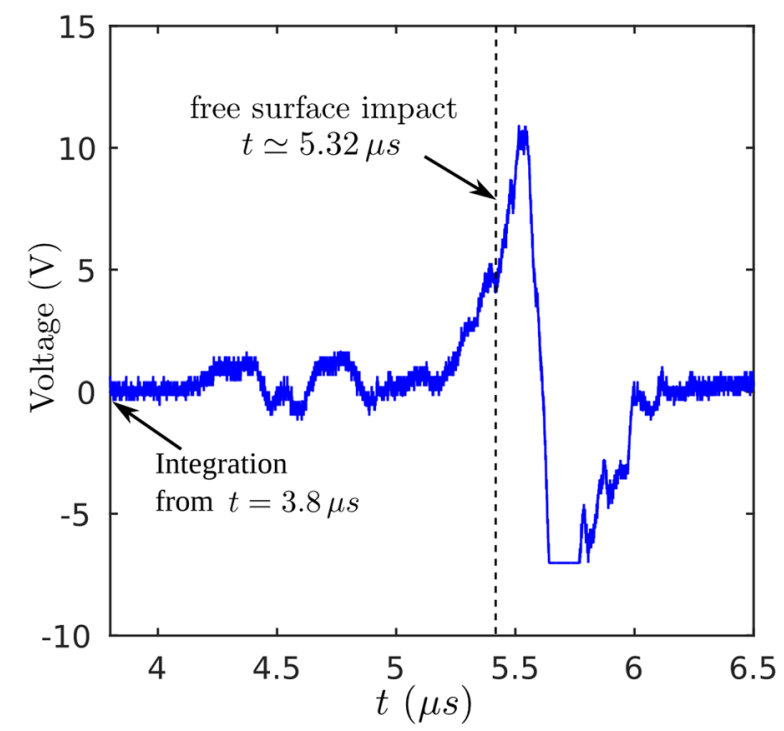

FIG. 11. LN-pin voltage $U(t)(\mathrm{V})$, located at $7 \mathrm{~mm}$ from the sample.

where $V_{\mathrm{p}}(t)(\mathrm{m} / \mathrm{s})$ are particle velocities. The expression of $M_{a}(t)$ as a function of velocity $V$ is called $M(V)$. At $V=V_{\mathrm{fs}}$, $M\left(V=V_{\mathrm{fs}}\right)=M_{s}$, the total areal mass of ejecta.

In Fig. 12, both pressure on the pin and integrated areal mass versus velocity are depicted. With $V_{\mathrm{fs}} \simeq 2013 \mathrm{~m} / \mathrm{s}$ (first experiment), the total areal mass is $M\left(V_{\mathrm{fs}}\right)=M_{\mathrm{s}} \simeq 7.3 \mathrm{mg} / \mathrm{cm}^{2}$.

\section{Maximum likelihood estimation}

To make comparison between experiment and simulations, we make the assumption of a power-law size distribution with exponent ${ }^{18,29} \gamma_{\mathrm{d}}=5.6$. Model 2 described by Eq. (21) is parametric and will be used for simulating average PDV spectra $\langle\Phi(V, t)\rangle_{t_{\infty}}$ with the given cloud's characteristics $\left(\gamma_{d}, \gamma_{v}\right.$, etc.). To the extent that a simple visual comparison is not relevant, we perform a Maximum Likelihood (ML) estimation of the parameters with our model. The purpose is to estimate the mass-velocity function $M(V)$ from PDV data. The model has been reduced to four parameters, two of whom with a real interest: the integrated areal mass density $M\left(V_{\min }\right)$ and the coefficient from the velocity distribution $\gamma_{\mathrm{v}}$. The third parameter is a multiplicative coefficient $\kappa$ and the fourth parameter is the background noise amplitude $\left\langle B_{\Phi}\right\rangle$. For a given point of the
PDV spectrogram $\left(V_{j}, t_{i}\right)$ in the absence of signal, the probability distribution of background noise $B_{\Phi_{\exp }}\left(V_{j}, t_{i}\right)$ (experimental results) is well approximated by a negative exponential,

$$
p\left(B_{\Phi_{\exp }}\left(V_{j}, t_{i}\right)\right)=\frac{1}{\left\langle B_{\Phi_{\text {exp }}}\right\rangle} \exp \left[-\frac{B_{\Phi_{\text {exp }}}\left(V_{j}, t_{i}\right)}{\left\langle B_{\Phi_{\text {exp }}}\right\rangle}\right] .
$$

If the background noise were absent $\left(\left\langle B_{\Phi_{\text {exp }}}\right\rangle=0\right)$, the distribution of independent values $\Phi_{\exp }\left(V, t_{i}\right)$ (experimental PDV spectrogram) at different times $t_{i}(i=1, \ldots, N)$ ( $N$ being the number of independent realizations from the PDV spectrogram) would follow a speckle statistics. The probability density function (PDF) would be

$$
p\left(\Phi_{\exp }\left(V, t_{i}\right)\right)=\frac{1}{\langle\Phi(V, t)\rangle_{t_{\infty}}} \exp \left[-\frac{\Phi_{\exp }\left(V, t_{i}\right)}{\langle\Phi(V, t)\rangle_{t_{\infty}}}\right] .
$$

To ensure the independence between $\Phi\left(V, t_{i}\right)$ and $\Phi\left(V, t_{j}\right)$ at constant velocity, the Fourier analysis on the raw PDV signal is made with a rectangular window (width $T_{w}=50 \mathrm{~ns}$, sliding time step $\delta T_{w}=T_{w}$, and no zero-padding). However, as background noise is present, we must correct Eq. (27) to take into account both the signal $\Phi_{\exp }\left(V, t_{i}\right)$ and average noise $\left\langle B_{\Phi_{\text {exp }}}\right\rangle$. When background noise is present, the $\operatorname{PDF} p\left(\Phi_{\exp }\left(V, t_{i}\right)\right)$ is the PDF of the sum of three random variables:

- $X=\Phi_{\exp }\left(V, t_{i}\right)$ whose PDF is given by Eq. (27),

- $Y=B_{\Phi_{\text {exp }}}\left(V_{j}, t_{i}\right)$ whose PDF is given by Eq. (26),

- and $Z=2 \cdot \sqrt{X} \cdot \sqrt{Y} \cos (\Theta)$, where $\Theta$ follows an uniform distribution between 0 and $2 \pi, \sqrt{X}$ and $\sqrt{Y}$ being described by two independent Rayleigh distributions.

We get,

$$
\begin{aligned}
p\left(\Phi_{\exp }\left(V, t_{i}\right)\right)= & \frac{1}{\left(\langle\Phi(V, t)\rangle_{t_{\infty}}+\left\langle B_{\Phi_{\text {exp }}}\right\rangle\right)} \\
& \times \exp \left[-\frac{\Phi_{\exp }\left(V, t_{i}\right)}{\left(\langle\Phi(V, t)\rangle_{t_{\infty}}+\left\langle B_{\left.\Phi_{\text {exp }}\right\rangle}\right\rangle\right)} .\right.
\end{aligned}
$$

For the experimental time-averaged PDV spectrum $\left\langle\Phi_{\exp }(V, t)\right\rangle_{t}$, the PDF becomes Gamma distributed ${ }^{30}$ (PDF of the mean of $N$ independent speckle realizations) and Eq. (28)
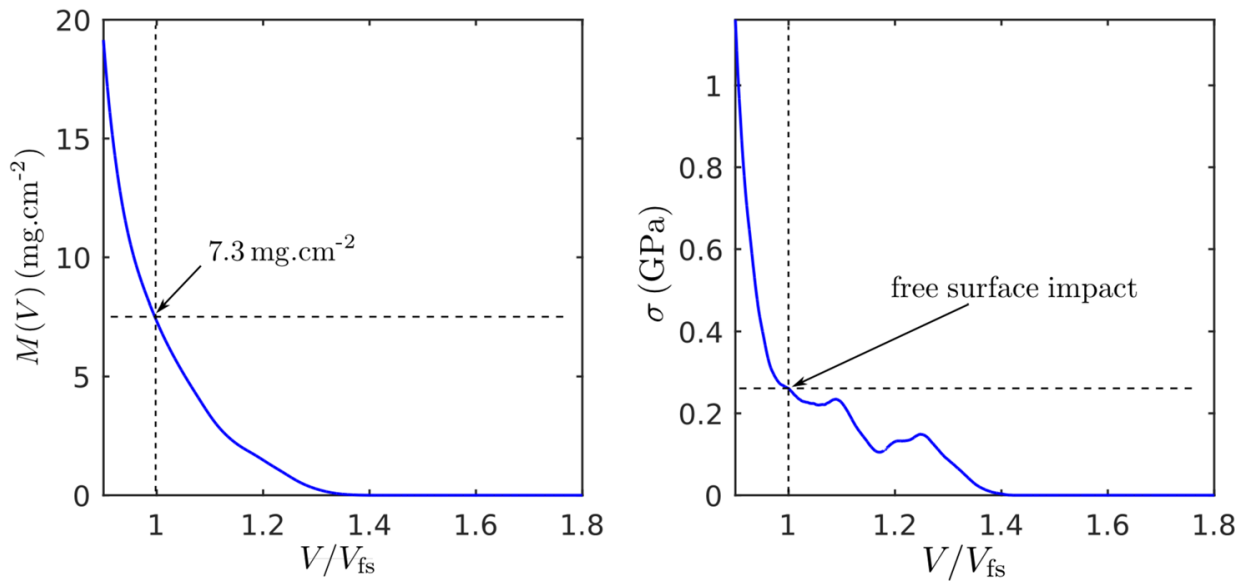

FIG. 12. Cumulative areal mass $M(V)$ $\left(\mathrm{mg} / \mathrm{cm}^{2}\right)$ and applied stress $\sigma(\mathrm{GPa})$ on the piezoelectric probe versus reduced velocity $V / V_{\mathrm{fs}}$ with $V_{\mathrm{fs}}=2013 \mathrm{~m} / \mathrm{s}$, determined from the first experiment. 


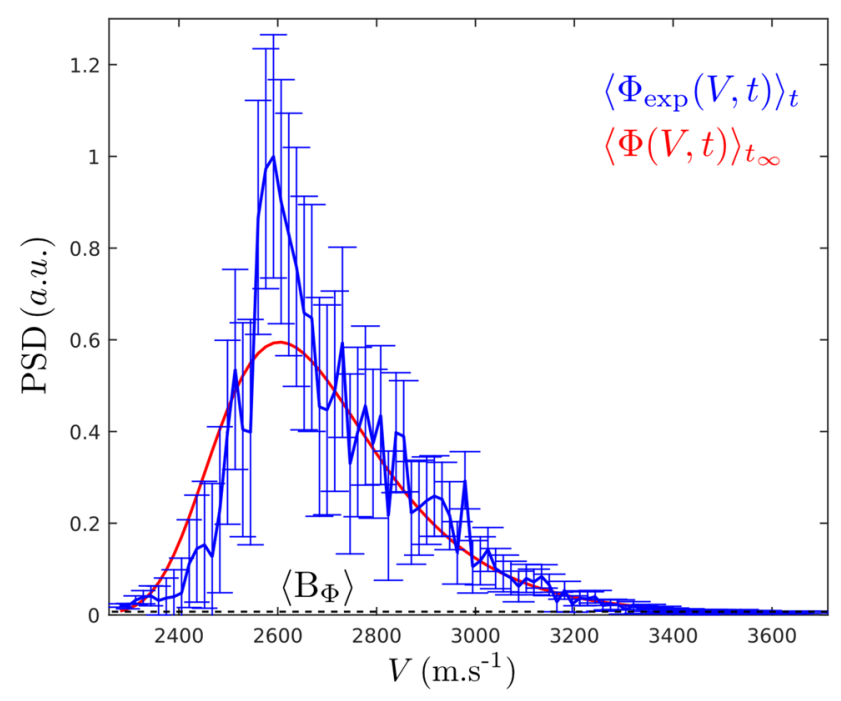

FIG. 13. Comparison between the normalized experimental time-averaged PDV data $\left\langle\Phi_{\exp }(V, t)\right\rangle_{t}$ (blue) and the PDV spectrum $\langle\Phi(V)\rangle_{t_{\infty}}$ obtained from the ML estimation: $\zeta_{o}=\left[M(V=2281 \mathrm{~m} / \mathrm{s})=2.05 \mathrm{mg} / \mathrm{cm}^{2}, \gamma_{\mathrm{v}}=23.28\right.$, $\left.\kappa=6.6 \times 10^{-3},\left\langle B_{\Phi}\right\rangle=2.8 \times 10^{-3}\right]$ (red), for $V$ between $2281 \mathrm{~m} / \mathrm{s}$ and $3676 \mathrm{~m} / \mathrm{s}$. Fourier parameters: Rectangular window $\left(T_{w}=50 \mathrm{~ns}, \delta T_{w}=T_{w}\right.$ without zeropadding). 20 PDV independent slices between 1.95 and $2.9 \mu$ s were used to average the spectrum. The black dashed line is the estimated background noise amplitude $\left\langle B_{\Phi}\right\rangle$. The error bars $( \pm 2 \hat{\sigma}(V)$, standard deviation) are estimated from the experimental PDV spectrogram.

becomes

$$
\begin{aligned}
p\left(\left\langle\Phi_{\exp }(V, t)\right\rangle_{t}\right)= & \frac{N^{N}\left\langle\Phi_{\exp }(V, t)\right\rangle_{t}^{N-1}}{\Gamma(N)\left(\langle\Phi(V, t)\rangle_{t_{\infty}}+\left\langle B_{\Phi_{\exp }}\right\rangle\right)^{N}} \\
& \times \exp \left[-N \frac{\left\langle\Phi_{\exp }(V, t)\right\rangle_{t}}{\left(\langle\Phi(V, t)\rangle_{t_{\infty}}+\left\langle B_{\Phi_{\exp }}\right\rangle\right)}\right] .
\end{aligned}
$$

The first three parameters of $\zeta=\left[M\left(V_{\min }\right), \gamma_{\mathrm{v}}, \kappa,\left\langle B_{\Phi}\right\rangle\right]$ are hidden in $\langle\Phi(V, t)\rangle_{t_{\infty}}=\left\langle\Phi\left(V, t, M\left(V_{\min }\right), \gamma_{\mathrm{v}}, \kappa\right)\right\rangle_{t_{\infty}} . \Gamma$ is the Gamma function. $M\left(V_{\min }\right)$ will be denoted as $M_{\mathrm{Vmin}}$. The goal is to find the maximum of the likelihood function defined by

$$
\begin{aligned}
& \mathcal{L}:(\left.\kappa, \gamma_{\mathrm{v}}, M_{\mathrm{V} \min },\left\langle B_{\Phi}\right\rangle\right) \\
& \mapsto \prod_{k=1}^{N_{\mathrm{V}}} \frac{N^{N}\left\langle\Phi_{\exp }\left(V_{k}, t\right)\right\rangle_{t}^{N-1}}{\Gamma(N)\left(\left\langle\Phi\left(V_{k}, t, \kappa, \gamma_{\mathrm{v}}, M_{\mathrm{Vmin}}\right)\right\rangle_{t_{\infty}}+\left\langle B_{\Phi}\right\rangle\right)^{N}} \\
& \quad \times \exp \left[-\frac{N\left\langle\Phi_{\exp }\left(V_{k}, t\right)\right\rangle_{t}}{\left\langle\left(\Phi\left(V_{k}, t, \kappa, \gamma_{\mathrm{v}}, M_{\mathrm{V} \min }\right)\right\rangle_{t_{\infty}}+\left\langle B_{\Phi}\right\rangle\right)}\right] .
\end{aligned}
$$

$N_{V}=101$ is the number of points extracted from the spectrogram, between $V_{k=1}=V_{\min }=2281.3 \mathrm{~m} / \mathrm{s}$ and $V_{k=101}$ $=V_{\max }=3676 \mathrm{~m} / \mathrm{s}$, and $N=20$ is the number of independent temporal PDV slices. A Raphson-Newton algorithm is used to minimize the negative log-likelihood function. The set of parameters, which is the most likely to have generated experimental PDV data, is $\zeta_{o}=\left[M_{\mathrm{V} \min }, \gamma_{\mathrm{v}}, \kappa,\left\langle B_{\Phi}\right\rangle\right]=\left[2.05 \mathrm{mg} / \mathrm{cm}^{2}\right.$, 23.28, $\left.6.6 \times 10^{-3}, 2.8 \times 10^{-3}\right]$.

In Fig. 13, both experimental time-averaged PDV spectrogram $\left\langle\Phi_{\exp }(V, t)\right\rangle_{t}$ and average PDV spectrogram $\langle\Phi(V, t)\rangle_{t_{\infty}}$ obtained from the ML estimation are depicted. The shape of the PDV spectrogram is well reproduced by our model, taking into account both increasing PSD related to particle densities in the cloud and decreasing PSD due to the scatter-induced attenuation in the low velocity region. For each velocity, the blue error bars are estimated from the experimental PDV spectrogram, $\hat{\sigma}(V)=\left(\left\langle\Phi_{\exp }(V, t)\right\rangle_{t} / \sqrt{N}\right)$ on $N=20$ uncorrelated points. Around $V=2600 \mathrm{~m} / \mathrm{s}$, the strong peak could be explained by local density fluctuations in the particle cloud, the standard deviation being underestimated by the Gamma
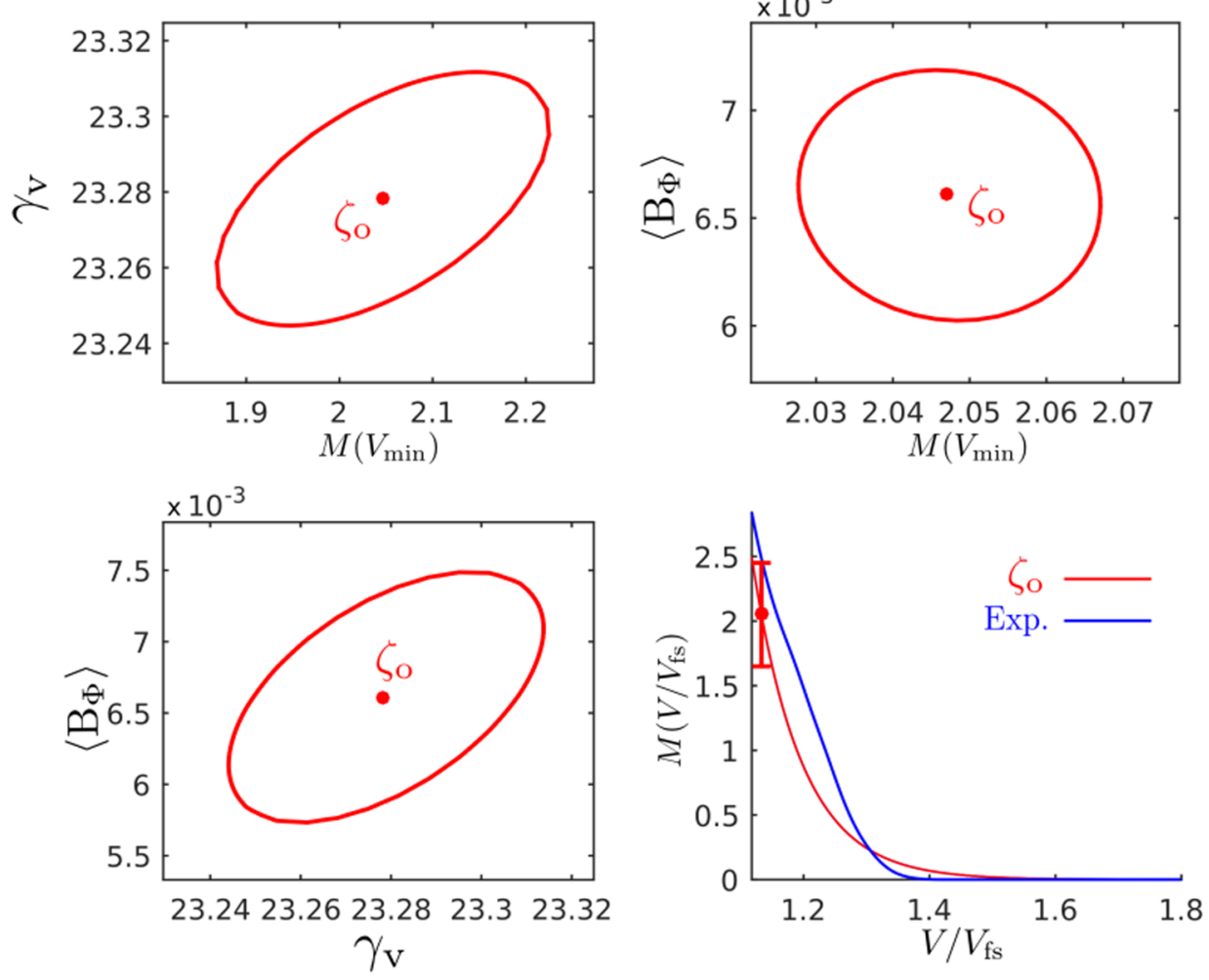

FIG. 14. Top left, top right, and bottom left: Projections of the constant likelihood function $\mathcal{L}(\zeta)$ such that the equality of Eq. (31) is verified (joint confidence region at $95 \%) . M\left(V_{\min }\right)$ has units of $\mathrm{mg} / \mathrm{cm}^{2}$. Scatter plot: ML estimation. Bottom right: Comparison between experimental results and ML estimation of the mass-velocity distribution $M(V)\left(\mathrm{mg} / \mathrm{cm}^{2}\right)$ for $V / V_{\mathrm{fs}}$ between 1.11 and 1.8. The error bar at $\pm 2 \sigma$ and $V_{\min } / V_{\mathrm{fs}}=1.13$ is calculated using the likelihood ratio method (boundaries of $\mathcal{L}$ ). 
density function of Eq. (29). The joint confidence region for the set of parameters $\left[M(V), \gamma_{\mathrm{v}}, \kappa,\left\langle B_{\Phi}\right\rangle\right]$ is a four-dimensional volume in the space of parameters and is estimated with the Likelihood Ratio (LR) method, $2 \log \left(\frac{\mathcal{L}\left(\zeta_{0}\right)}{\mathcal{L}(\zeta)}\right)$ being approximated as a $\chi_{4}^{2}(1-\alpha)$ with $\alpha=0.05$ (confidence region of $95 \%$ ),

$$
\left\{\zeta: 2 \log \left(\frac{\mathcal{L}\left(\zeta_{o}\right)}{\mathcal{L}(\zeta)}\right) \leq \chi_{4}^{2}(1-\alpha)\right\}
$$

In Fig. 14, three projections of a constant likelihood function are provided to illustrate the joint confidence region for the set of parameters $\zeta$, such that $\mathcal{L}(\zeta)=\mathcal{L}\left(\zeta_{o}\right) \exp \left[-\frac{1}{2} \chi_{4}^{2}(1-\alpha)\right] . \kappa$ and $\left\langle B_{\Phi}\right\rangle$ have a small impact on the estimation. On the contrary, the main uncertainty on $M_{V_{\min }}$ comes from the coupling of crucial importance between $M_{V_{\min }}$ and $\gamma_{\mathrm{v}}$. The integrated mass-velocity function $M(V)$ (bottom right) is deduced from the two parameters $M_{\mathrm{Vmin}}$ and $\gamma_{\mathrm{v}}$ using

$$
M(V)=M_{\mathrm{V} \min } \exp \left[-\gamma_{\mathrm{v}} \frac{\left(V-V_{\min }\right)}{V_{\max }}\right] .
$$

A good agreement is obtained between the ML estimation and the experimental results $\left[M_{V_{\min }}=2.05 \pm 0.36 \mathrm{mg} / \mathrm{cm}^{2}\right.$ for the ML estimation and $M\left(V_{\min } \simeq 2.48 \mathrm{mg} / \mathrm{cm}^{2}\right)$ for experimental LN-pin data]. It appears that for $V / V_{\mathrm{fs}}>1.3$, the LN-pin does not detect the presence of high-speed ejecta particles. In this case, an estimate from PDV data is relevant. Further comparisons between model 2 [Eq. (21)] and experimental PDV data, for which a time-averaging of the spectrum can be done over a larger interval, are of great interest. If the number of points used to average the spectrum is large, the probability density function of the averaged spectrum will be well approximated by a normal distribution and the ML estimate will converge with which it is obtained from a non-linear least square minimization. Furthermore, since the joint confidence intervals will be reduced, the estimation will be improved. Our

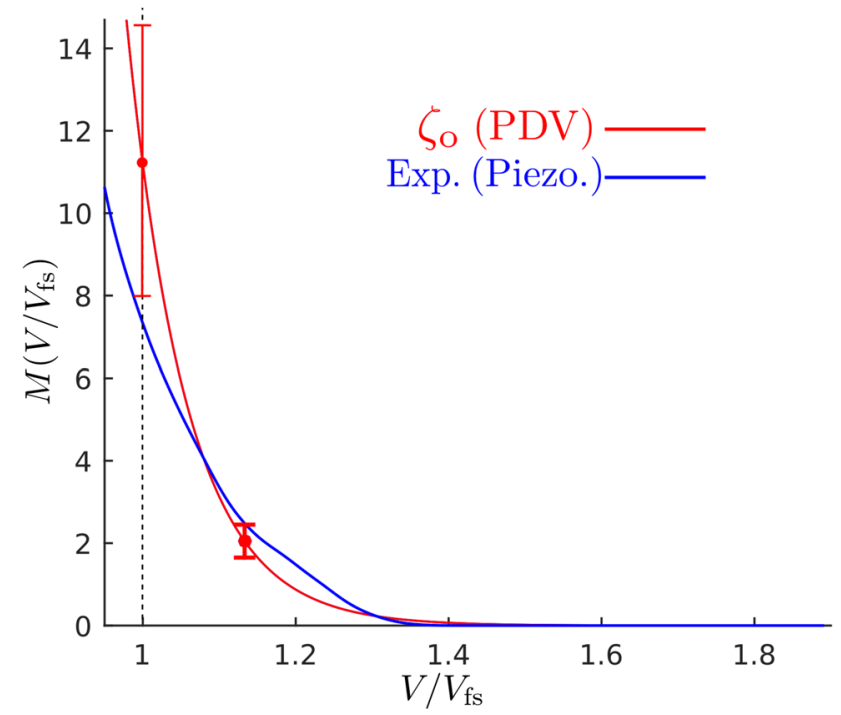

FIG. 15. Comparison between the ML estimate $M(V)\left(\mathrm{mg} \mathrm{cm}^{-2}\right)$ performed on experimental PDV data and experimental $M(V)$ measured with a piezoelectric LN-pin, for $V / V_{\mathrm{fs}}$ between 1 and 1.8. The error bar at $\pm 2 \sigma$ and $V / V_{\mathrm{fs}}=1$ is estimated from the uncertainty determined at $V / V_{\mathrm{fs}}=1.13$ using the likelihood ratio method (boundaries of $\mathcal{L}$ ). estimation from PDV data being limited to $V_{\min }=2281 \mathrm{~m} / \mathrm{s}$, an extrapolation of $M(V)$ to smaller velocities is possible but uncertain (no information gain). In our case, $k h=0.42$ ( $k=2 \pi / 60 \mu \mathrm{m}^{-1}$ and $h=4 \mu \mathrm{m}$ ) and an extrapolation using the exponential model of the mass-velocity seems therefore relevant. A total areal mass of $M_{\mathrm{s}}=11.2 \pm 3.4 \mathrm{mg} / \mathrm{cm}^{2}$ is obtained from our PDV-based analysis, compared to LN-pin result $M_{\mathrm{S}}=7.3 \mathrm{mg} / \mathrm{cm}^{2}$ (see Fig. 15).

\section{DISCUSSION}

Section IV discusses how the estimate is modified when various assumptions are violated and how combining different diagnostics may improve the analysis.

\section{A. Random particle arrangement}

In this article, a random particle arrangement is assumed and fully justified by the speckle nature of the PDV spectrogram, $\langle\Phi(V, t)\rangle_{t_{\infty}}$ being described by the negative exponential distribution of a random phasor. The following relationship can be used to test whether or not experimental results follow this distribution:

$$
\left\langle\Phi_{\exp }^{N}(V, t)\right\rangle_{t}=\left\langle\Phi_{\exp }(V, t)\right\rangle_{t}^{N} N ! .
$$

This relation has been verified up to $N=4$ for the experimental results shown in Fig. 13.

\section{B. Particle shapes}

The average extinction efficiency $\overline{\mathbf{Q}_{\mathrm{ext}}}$ is determined using the Mie theory only valid for spherical particles. In practice, particles are not perfectly spherical and ellipsoidal shapes may be present in the ejecta. An extended Mie scattering theory or any electromagnetic solver can be used to study how the analysis might be affected. For randomly oriented ellipsoidal particles with respect to the PDV beam, the extinction efficiency will not be drastically modified for aspect ratios (the ratio between the length and the width of the particle) between 1 (sphere) and 2. We believe that assuming particles' sphericity is reasonable and introducing ellipsoidal shapes will unnecessarily increase the complexity of the problem for a first order theory of scattering in the ejecta cloud.

\section{Particle sizes}

In this article, a power law dependence with exponent $\gamma_{\mathrm{d}}=5.6$ has been assumed, between $d_{\min }=1 \mu \mathrm{m}$ and $d_{\max }$ $=10 \mu \mathrm{m}$. Since most of the mass is present in the region near $d_{\text {min }}$, a precise knowledge of the lower bound of the distribution is of paramount importance. Since it is likely that submicron particles are present in the ejecta, the impact of lowering $d_{\text {min }}$ down to $0.3 \mu \mathrm{m}$ must be studied. We also analyze how the areal mass estimate is modified when the critical exponent is lowered down to $\gamma_{\mathrm{d}}=3.4$.

The impact of lowering $d_{\min }$ is shown in Fig. 16. Down to $0.5 \mu \mathrm{m}$, the estimated areal mass $M_{s}$ decreases almost linearly. For $d_{\min }=0.3 \mu \mathrm{m}, M_{s}$ increases up to $8 \mathrm{mg} / \mathrm{cm}^{2}$. In this region, extinction cross sections are so small that their contribution to the extinction of light is reduced. On the contrary, their 


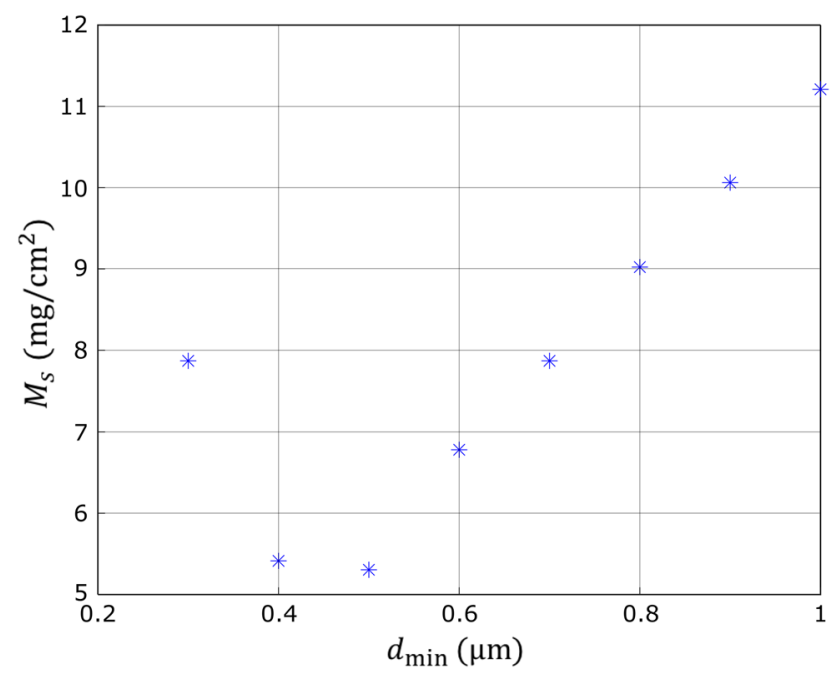

FIG. 16. Influence of lowering $d_{\text {min }}$ on the estimated areal mass $M_{s}$ from PDV data (power law particle size distribution with exponent $\gamma_{\mathrm{d}}=5.6$ ).

contribution to the total mass of the cloud is not negligible. The evolution of the estimated areal mass $M_{s}$ versus $\gamma_{\mathrm{d}}$ is shown in Fig. 17. The decrease is mostly attributed to an increased average particle diameter in the ejecta cloud.

The analysis will drastically be improved by a precise knowledge of the size distribution. Indeed, the inversion from PDV data is severely under-constrained and the data will never be inverted if a particle size distribution is not assumed (the consistency of the ML estimator would be lost). Further experimental investigations are needed to relax this strong assumption. A log-normal distribution may also be assumed for the size distribution: ${ }^{31}$

$$
f_{\mathrm{d}}\left(d_{\mathrm{p}}\right) \propto \frac{1}{d_{\mathrm{p}} \sigma \sqrt{2 \pi}} e^{-\frac{\left(\log d_{\mathrm{p}}-\log \mu\right)^{2}}{2 \sigma^{2}}}
$$

For $\mu=2$ and $\sigma=0.1,0.2$, and $0.5, M_{s}$ are, respectively, equal to $13.9,14.3$, and $19.3 \mathrm{mg} / \mathrm{cm}^{2}$. For $\mu=3$ and the same values

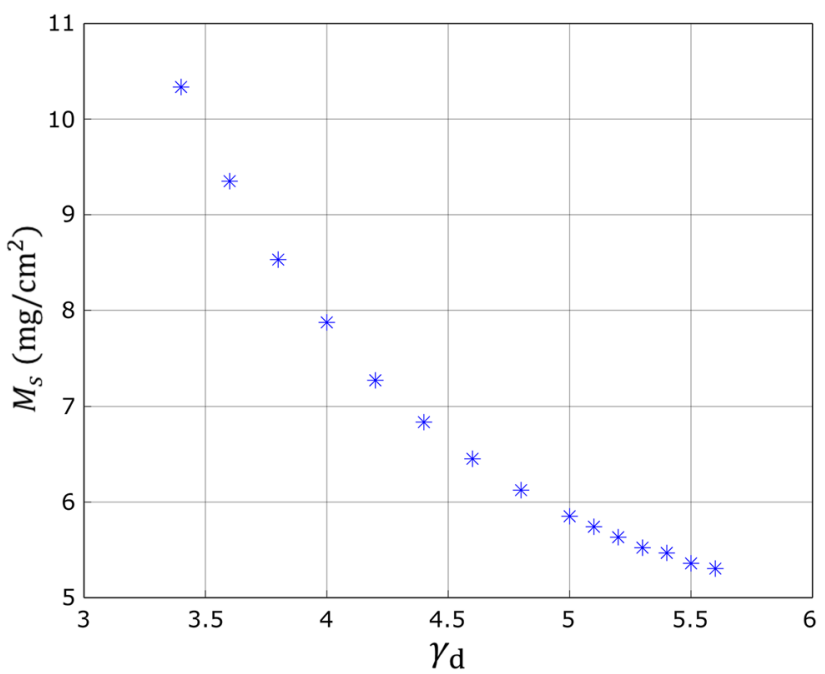

FIG. 17. Influence of lowering $\gamma_{\mathrm{d}}$ on the estimated areal mass $M_{s}$ from PDV data (power law particle size distribution with exponent $\gamma_{\mathrm{d}}$ ). $d_{\min }=0.5 \mu \mathrm{m}$ and $d_{\max }=10 \mu \mathrm{m}$. for $\sigma$, we get 21.2 , and 22.2 and $28.4 \mathrm{mg} / \mathrm{cm}^{2}$. The increased estimate is justified by the reduced number of small particles (tail of the log-normal distribution) in the cloud.

\section{Multiple scattering}

The first order solution presented in this paper does not take into account multiply scattered photons. For the highvelocity region of the spectrum, they may contribute ${ }^{17}$ at second order in the formation of the Doppler signal. The discrepancy between our model and experimental results observed around $2600 \mathrm{~m} / \mathrm{s}$ in Fig. 13 may also be explained by the multiple scattering of light in the ejecta cloud. After the first scattering event, the photon can be preferentially scattered in the forward direction and will not acquire any Doppler shift since incoming and scattered directions will be identical. If this event takes place in the high-velocity region of the cloud above $2600 \mathrm{~m} / \mathrm{s}$, a second scattering may eventually occur in the back direction around $2600 \mathrm{~m} / \mathrm{s}$, increasing the PSD in this region with respect to the amplitude obtained using a first order theory of scattering. Further investigations are deemed necessary to validate this assumption. The general method described in this paper can be applied to other models of average PDV spectrum $\langle\Phi(V, t)\rangle_{t_{\infty}}$ for which more physics is taken into account.

\section{E. Combining different diagnostics}

Additional diagnostics are needed to improve the inversion. An advantage of PDV inversion is that the retrieved areal mass depends neither on the time of shock breakout nor on the distance between the PDV probe and the cloud (for a collimated beam). Asay foil, X-ray absorption and piezoeletric pins diagnostics can be used to measure the massvelocity distribution in the ejecta cloud. In this case, the massvelocity function can be related to the velocity distribution $M(V) \propto \int_{V}^{V_{\max }} f_{\mathrm{v}}(V) \mathrm{d} V$. Different particle size distributions can accordingly be tested to find out which one is likely to explain experimental PDV results. Further experiments using Asay foil diagnostic will be very relevant and will certainly give more information on the shape of the mass-velocity distribution (the assumption of an exponential function will be relaxed). Small Asay foils ${ }^{32}$ may be implemented nearest to a PDV probe such that the two diagnostics will look at the same region of the ejecta. As already discussed in Sec. IV C, any particle sizing diagnostic will also drastically improve the analysis.

\section{CONCLUSION}

In this article, we have developed two models to study the way how the properties of an ejecta cloud expanding in a vacuum influence the shape of the time-velocity PDV spectrogram. The first one accounts for the speckle fluctuations observed on PDV data and the second one describes an average spectrum which would be obtained from averaging an infinite number of realizations. This model [Eq. (21)] is used to study the influence of the areal mass, the size and the velocity distributions. A comparison between the time-averaged PDV spectrum measured on a shock-loaded tin plate experiment and our model is presented, showing promising comparisons 
between experiment and simulation. A maximum likelihood analysis was done to retrieve the properties of the cloud, and an integrated areal mass of $M\left(V / V_{\mathrm{fs}}=1.13\right)=2.05 \pm 0.36 \mathrm{mg} / \mathrm{cm}^{2}$ is deduced, in line with piezoelectric pin result $M\left(V / V_{\mathrm{fs}}=1.13\right)$ $\simeq 2.48 \mathrm{mg} / \mathrm{cm}^{2}$, showing that correlations between piezoelectric and PDV diagnostics are possible. The total areal mass estimated from PDV is $M_{\mathrm{s}}=11.2 \pm 3.4 \mathrm{mg} / \mathrm{cm}^{2}$. This analysis also highlights the limited depth probed by the PDV beam in the particle cloud: below a given velocity $\left(V_{\min } \simeq 2281 \mathrm{~m} / \mathrm{s}\right)$, the optical intensity is too low and the signal to noise ratio falls drastically, limiting the range of the analysis. A future study will focus on both improving the depth probed by the beam and relaxing the strong hypothesis on particle sizes. Finding the role of particle cloud's properties on PDV spectra is a first step to solve the inversion of the problem, and correlations between different diagnostics will be necessary to reduce the number of solutions and improve the analysis. We believe that PDV may generate valuable data for an areal mass estimation of ejecta particles.

${ }^{1}$ M. B. Zellner, M. Grover, J. E. Hammerberg, R. S. Hixson, A. J. Iverson, G. S. Macrum, K. B. Morley, A. W. Obst, R. T. Olson, J. R. Payton, P. A. Rigg, N. Routley, G. D. Stevens, W. D. Turley, L. Veeser, and W. T. Buttler, "Effects of shock-breakout pressure on ejection of micron-scale material from shocked tin surfaces," J. Appl. Phys. 102, 013522 (2007).

${ }^{2}$ M. B. Zellner, W. V. McNeil, J. E. Hammerberg, R. S. Hixson, A. W. Obst, R. T. Olson, J. R. Payton, P. A. Rigg, N. Routley, G. D. Stevens, W. D. Turley, L. Veeser, and W. T. Buttler, "Probing the underlying physics of ejecta production from shocked Sn samples," J. Appl. Phys. 103, 123502 (2008).

${ }^{3}$ W. T. Buttler, D. M. Oró, D. L. Preston, K. O. Mikaelian, F. J. Cherne, R. S. Hixson, F. G. Mariam, C. Morris, J. B. Stone, G. Terrones, and D. Tupa, "Unstable Richtmyer-Meshkov growth of solid and liquid metals in vacuum," J. Fluid Mech. 703, 60-84 (2012).

${ }^{4}$ O. Durand and L. Soulard, "Large-scale molecular dynamics study of jet breakup and ejecta production from shock-loaded copper with a hybrid method," J. Appl. Phys. 111, 044901 (2012).

${ }^{5}$ O. Durand and L. Soulard, "Power law and exponential ejecta size distributions from the dynamic fragmentation of shock-loaded $\mathrm{Cu}$ and $\mathrm{Sn}$ metals under melt conditions," J. Appl. Phys. 114, 194902 (2013).

${ }^{6}$ H. An-Min, W. Pei, S. Jian-Li, and D. Su-Qing, "Molecular dynamics simulations of jet breakup and ejecta production from a grooved $\mathrm{Cu}$ surface under shock loading," Chin. Phys. B 23, 047102 (2014).

${ }^{7}$ P. Mercier, J. Bénier, A. Azzolina, J. Lagrange, and D. Partouche, "Photonic Doppler velocimetry in shock physics experiments," J. Phys. IV 134, 805-812 (2006).

${ }^{8}$ P. Mercier, J. Bénier, P. Frugier, G. Contencin, J. Veaux, S. Lauriot-Basseuil, and M. Debruyne, "Heterodyne velocimetry and detonics experiments," in 28th International Congress on High-Speed Imaging and Photonics (International Society for Optics and Photonics, 2008), p. 712610.

${ }^{9}$ C. Bizeuil, P. Chapron, P. Elias, and B. Laurent, "Experimental study of shock waves driven matter ejection on the free surface of a metal," Proc. SPIE 0491, 1030-1034 (1985).

${ }^{10}$ P. A. Rigg, W. W. Anderson, R. T. Olson, W. T. Buttler, and R. S. Hixson, "Investigation of ejecta production in tin using plate impact experiments," AIP Conf. Proc. 845, 1283 (2006).

${ }^{11}$ T. de Rességuier, L. Signor, A. Dragon, and G. Roy, "Dynamic fragmentation of laser shock-melted tin: Experiment and modelling," Int. J. Fract. 163, 109-119 (2010).

${ }^{12}$ T. de Rességuier, E. Lescoute, A. Sollier, G. Prudhomme, and P. Mercier, "Microjetting from grooved surfaces in metallic samples subjected to laser driven shocks," J. Appl. Phys. 115, 043525 (2014).
${ }^{13}$ S. K. Monfared, D. M. Oró, M. Grover, J. E. Hammerberg, B. M. LaLone, C. L. Pack, M. M. Schauer, G. D. Stevens, J. B. Stone, W. D. Turley, and W. T. Buttler, "Experimental observations on the links between surface perturbation parameters and shock-induced mass ejection," J. Appl. Phys. 116, 063504 (2014).

${ }^{14}$ J. Asay, L. Mix, and F. Perry, "Ejection of material from shocked surfaces," Appl. Phys. Lett. 29, 284-287 (1976).

${ }^{15}$ O. T. Strand, L. V. berzins, D. R. Goosman, W. W. Kuholow, P. D. Sargis, and T. L. Whitworth, "Velocimetry using heterodyne techniques," in 26th International Congress on High-Speed Photography and Photonics (International Society for Optics and Photonics, 2005), pp. 593-599.

${ }^{16}$ E. A. Moro, M. E. Briggs, and L. M. Hull, "Defining parametric dependencies for the correct interpretation of speckle dynamics in photon Doppler velocimetry," Appl. Opt. 52, 8661-8669 (2013).

${ }^{17}$ A. Andriyash, M. Astashkin, V. Baranov, A. Golubinskii, D. Irinichev, A. Kondrat'ev, S. Kuratov, V. MAzanov, D. Rogozkin, S. Stepushkin et al., "Optoheterodyne Doppler measurements of the ballistic expansion of the products of the shock wave-induced surface destruction: Experiment and theory," J. Exp. Theor. Phys. 122, 970-983 (2016).

${ }^{18}$ D. Sorenson, R. Minich, J. Romero, T. Tunnell, and R. Malone, "Ejecta particle size distributions for shock loaded Sn and Al metals," J. Appl. Phys. 92, 5830-5836 (2002).

${ }^{19}$ G. Prudhomme, P. Mercier, and L. Berthe, "PDV experiments on shockloaded particles," J. Phys.: Conf. Ser. 500, 142027 (2014).

${ }^{20}$ G. Prudhomme, P. Mercier, L. Berthe, J. Bénier, and P.-A. Frugier, "Frontal and tilted PDV probes for measuring velocity history of laser-shock induced calibrated particles," J. Phys.: Conf. Ser. 500, 142022 (2014).

${ }^{21}$ G. Prudhomme, "Étude des nuages de particules éjectées sous choc: Apports de la vélocimétrie hétérodyne," Ph.D. thesis, Arts et Métiers Paris Tech, 2014.

${ }^{22}$ C. F. Bohren and D. R. Huffman, Absorption and Scattering of Light by Small Particles (Wiley-VCH, Weinheim, 2004).

${ }^{23}$ O. Durand and L. Soulard, "Mass-velocity and size-velocity distributions of ejecta cloud from shock-loaded tin surface using atomistic simulations," J. Appl. Phys. 117, 165903 (2015).

${ }^{24} \mathrm{G}$. Dimonte and P. Ramaprabhu, "Simulations and model of the nonlinear Richtmyer-Meshkov instability," Phys. Fluids 22, 014104 (2010).

${ }^{25}$ G. Dimonte, G. Terrones, F. J. Cherne, and P. Ramaprabhu, "Ejecta source model based on the nonlinear Richtmyer-Meshkov instability," J. Appl. Phys. 113, 024905 (2013).

${ }^{26}$ A. Mears, N. R. Routley, and P. A. Kendall, "Impact stress measurement using piezoelectric probes with PZT and lithium niobate elements," AIP Conf. Proc. 1195, 639 (2009).

${ }^{27}$ W. S. Vogan, W. W. Anderson, M. Grover, J. E. Hammerberg, N. S. P. King, S. K. Lamoreaux, G. Macrum, K. B. Morley, P. A. Rigg, G. D. Stevens, W. D. Turley, L. R. Veeser, and W. T. Buttler, "Piezoelectric characterization of ejecta from shocked tin surfaces," J. Appl. Phys. 98, 113508 (2005).

${ }^{28}$ W. T. Buttler, M. B. Zellner, R. T. Olson, P. A. Rigg, R. S. Hixson, J. E. Hammerberg, A. W. Obst, J. R. Payton, A. Iverson, and J. Young, "Dynamic comparisons of piezoelectric ejecta diagnostics,"J. Appl. Phys. 101, 063547 (2007).

${ }^{29}$ D. Sorenson, R. Malone, B. Frogget, C. Ciarcia, T. Tunnell, and R. Flurer, "Particle distribution measurements using in-line fraunhofer holography," in 22nd International Congress on High-Speed Photography and Photonics (International Society for Optics and Photonics, 1997), pp. 206-213.

${ }^{30}$ J. W. Goodman, Speckle Phenomena in Optics: Theory and Applications (Roberts and Company, 2007).

${ }^{31}$ D. S. Sorenson, P. D. Pazuchanics, R. P. Johnson, T. Tunnell, D. Smalley, R. Malone, M. Kaufman, D. Marks, G. Capelle, M. Grover et al., "Ejecta particle size measurements from the break-up of micro-jets in vacuum and helium gas using ultraviolet in-line fraunhofer holography," AIP Conf. Proc. 1793, 100026 (2017).

${ }^{32}$ P. Steele, B. Jacoby, S. Compton, and J. Sinibaldi, "Advances in ejecta diagnostics at LLNL,” J. Dyn. Behav. Mater. 3, 253-264 (2017). 